\title{
Existence results for a coupled system of Caputo type fractional integro-differential equations with multi-point and sub-strip boundary conditions
}

\author{
Ahmed Alsaedi ${ }^{1}$, Amjad F. Albideewi ${ }^{1}$, Sotiris K. Ntouyas ${ }^{2,1}$ and Bashir Ahmad ${ }^{1 *}$ (D
}

\section{"Correspondence:}

bashirahmad_qau@yahoo.com

${ }^{1}$ Nonlinear Analysis and Applied

Mathematics (NAAM) - Research Group, Department of Mathematics,

Faculty of Science, King Abdulaziz University, P.O. Box 80203, Jeddah

21589, Saudi Arabia

Full list of author information is

available at the end of the article

\section{Springer}

\begin{abstract}
This paper is concerned with the existence and uniqueness of solutions for a coupled system of Liouville-Caputo type fractional integro-differential equations with multi-point and sub-strip boundary conditions. The fractional integro-differential equations involve Caputo derivative operators of different orders and finitely many Riemann-Liouville fractional integral and non-integral type nonlinearities. The boundary conditions at the terminal position $t=1$ involve sub-strips and multi-point contributions. The Banach fixed point theorem and the Leray-Schauder alternative are used to establish our results. The obtained results are illustrated with the aid of examples.
\end{abstract}

MSC: $34 \mathrm{~A} 08 ; 34 \mathrm{~B} 10 ; 34 \mathrm{~B} 15$

Keywords: Caputo derivative; Riemann-Liouville integral; Coupled system; Multi-point boundary conditions; Existence; Fixed point theorem

\section{Introduction}

Fractional calculus has evolved as an important area of investigation owing to its extensive applications in natural and social sciences. Examples include bio-engineering [1], ecology [2], financial economics [3], chaos and fractional dynamics [4], etc. The tools of fractional calculus have improved the mathematical modeling of many real-world problems [5-7]. It has been mainly due to the nonlocal nature of fractional-order differential and integral operators. Fractional-order mathematical models often consist of coupled systems of fractional-order differential and integro-differential equations. For theoretical treatment of such systems, we refer the reader to the papers [8-19] and the references cited therein. In [20], a fractional-order nonlinear mixed coupled system with coupled integrodifferential boundary conditions was studied. In a recent article [21], the authors investigated the existence of solutions for the systems of Caputo and Riemann-Liouville type mixed-order coupled fractional differential equations and inclusions equipped with with coupled integral fractional boundary conditions.

(c) The Author(s) 2021. This article is licensed under a Creative Commons Attribution 4.0 International License, which permits use, sharing, adaptation, distribution and reproduction in any medium or format, as long as you give appropriate credit to the original author(s) and the source, provide a link to the Creative Commons licence, and indicate if changes were made. The images or other third party material in this article are included in the article's Creative Commons licence, unless indicated otherwise in a credit line to the material. If material is not included in the article's Creative Commons licence and your intended use is not permitted by statutory regulation or exceeds the permitted use, you will need to obtain permission directly from the copyright holder. To view a copy of this licence, visit http://creativecommons.org/licenses/by/4.0/. 
In the present study, we investigate a new class of nonlinear coupled systems of Liouville-Caputo type fractional integro-differential equations

$$
\begin{cases}{ }^{c} D^{q} x(t)+\sum_{i=1}^{k} I^{p_{i}} g_{i}(t, x(t), y(t))=f_{1}(t, x(t), y(t)), & 1<q \leq 2, t \in[0,1], \\ { }^{c} D^{\delta} y(t)+\sum_{j=1}^{l} I^{v_{j}} h_{j}(t, x(t), y(t))=f_{2}(t, x(t), y(t)), & 1<\delta \leq 2, t \in[0,1]\end{cases}
$$

subject to the boundary conditions

$$
\left\{\begin{array}{l}
x(0)=a_{1}, \quad y(0)=b_{1}, \\
\alpha_{1} x(1)+\beta_{1} x^{\prime}(1)=\gamma_{1} \int_{0}^{\zeta} y(s) d s+\sum_{m=1}^{\omega} \mu_{m} y\left(\eta_{m}\right), \\
\alpha_{2} y(1)+\beta_{2} y^{\prime}(1)=\gamma_{2} \int_{0}^{\zeta} x(s) d s+\sum_{m=1}^{\omega} \xi_{m} x\left(\eta_{m}\right),
\end{array}\right.
$$

where ${ }^{c} D^{q},{ }^{c} D^{\delta}$ respectively denote the Caputo fractional derivative operators of order $q, \delta \in(1,2]$ and $f_{1}, f_{2}, g_{i}, h_{j}:[0,1] \times \mathbb{R} \times \mathbb{R} \rightarrow \mathbb{R}(i=1, \ldots, k)(j=1, \ldots, l)$ are continuous functions, $a_{1}, b_{1}, \alpha_{1}, \alpha_{2}, \beta_{1}, \beta_{2}, \gamma_{1}, \gamma_{2}, \mu_{m}, \xi_{m} \in \mathbb{R}$ and $\zeta, \eta_{m} \in(0,1), m=1,2, \ldots, \omega$.

Existence and uniqueness results for the given problem are established via Banach fixed point theorem and Leray-Schauder alternative. The main results are presented in Sect. 3. In Sect. 2, some basic definitions from fractional calculus are recalled and an auxiliary result concerning the linear version of problem (1)-(2), which is essential to define the solution of problem (1)-(2), is proved. Examples illustrating the obtained results are constructed in Sect. 4.

\section{Preliminaries}

Let us recall some basic definitions on fractional calculus [22].

Definition 1 For a function $g \in A C^{n}[a, b]$, the Caputo derivative of fractional order $q \in$ $(n-1, n], n \in \mathbb{N}$, existing almost everywhere on $[a, b]$, is defined as follows:

$$
{ }^{c} D^{q} g(t)=\frac{1}{\Gamma(n-q)} \int_{a}^{t}(t-s)^{n-q-1} g^{(n)}(s) d s, \quad t \in[a, b] .
$$

Definition 2 The Riemann-Liouville fractional integral of order $q>0$ for $g \in L_{1}[a, b]$, existing almost everywhere on $[a, b]$, is defined as

$$
I^{q} g(t)=\frac{1}{\Gamma(q)} \int_{a}^{t} \frac{g(s)}{(t-s)^{1-q}} d s, \quad t \in[a, b] .
$$

Lemma 1 For $m-1<q \leq m$, the general solution of the fractional differential equation ${ }^{c} D^{q} x(t)=0$ is given by

$$
x(t)=c_{0}+c_{1} t+c_{2} t^{2}+\cdots+c_{m-1} t^{m-1}
$$

where $c_{i} \in \mathbb{R}, i=0,1,2, \ldots, m-1$.

In view of Lemma 1, it follows that

$$
I^{q c} D^{q} x(t)=x(t)+c_{0}+c_{1} t+c_{2} t^{2}+\cdots+c_{m-1} t^{m-1}
$$

for some $c_{i} \in \mathbb{R}, i=0,1,2, \ldots, m-1$. 
To study nonlinear problem (1), we first consider the associated linear problem and obtain its solution.

Lemma 2 Let $\Lambda_{1} \neq 0$. For $\widehat{f_{1}}, \widehat{f_{2}} \in C([0,1], \mathbb{R})$ the integral solution of the linear system of fractional differential equations

$$
\begin{cases}{ }^{c} D^{q} x(t)=\widehat{f_{1}}(t), & 1<q \leq 2, t \in[0,1], \\ { }^{c} D^{\delta} y(t)=\widehat{f_{2}}(t), & 1<\delta \leq 2, t \in[0,1],\end{cases}
$$

supplemented with the boundary conditions (2) is given by

$$
\begin{aligned}
x(t)= & \int_{0}^{t} \frac{(t-s)^{q-1}}{\Gamma(q)} \widehat{f_{1}}(s) d s+a_{1}-\frac{t}{\Lambda_{1}}\left[\sigma_{1} \int_{0}^{1} \frac{(1-s)^{q-1}}{\Gamma(q)} \widehat{f}_{1}(s) d s\right. \\
& +\sigma_{2} \int_{0}^{1} \frac{(1-s)^{q-2}}{\Gamma(q-1)} \widehat{f_{1}}(s) d s-\sigma_{3} \int_{0}^{\zeta} \int_{0}^{s} \frac{(s-u)^{\delta-1}}{\Gamma(\delta)} \widehat{f_{2}}(u) d u d s \\
& -\sigma_{4} \int_{0}^{\eta_{m}} \frac{\left(\eta_{m}-s\right)^{\delta-1}}{\Gamma(\delta)} \widehat{f}_{2}(s) d s+\sigma_{5} \int_{0}^{1} \frac{(1-s)^{\delta-1}}{\Gamma(\delta)} \widehat{f_{2}}(s) d s \\
& +\sigma_{6} \int_{0}^{1} \frac{(1-s)^{\delta-2}}{\Gamma(\delta-1)} \widehat{f}_{2}(s) d s-\sigma_{7} \int_{0}^{\zeta} \int_{0}^{s} \frac{(s-u)^{q-1}}{\Gamma(q)} \widehat{f}_{1}(u) d u d s \\
& \left.-\sigma_{8} \int_{0}^{\eta_{m}} \frac{\left(\eta_{m}-s\right)^{q-1}}{\Gamma(q)} \widehat{f_{1}}(s) d s+\Lambda_{2}\right]
\end{aligned}
$$

and

$$
\begin{aligned}
y(t)= & \int_{0}^{t} \frac{(t-s)^{\delta-1}}{\Gamma(\delta)} \widehat{f_{2}}(s) d s+b_{1}-\frac{t}{\Lambda_{1}}\left[\sigma_{9} \int_{0}^{1} \frac{(1-s)^{q-1}}{\Gamma(q)} \widehat{f_{1}}(s) d s\right. \\
& +\sigma_{10} \int_{0}^{1} \frac{(1-s)^{q-2}}{\Gamma(q-1)} \widehat{f_{1}}(s) d s-\sigma_{11} \int_{0}^{\zeta} \int_{0}^{s} \frac{(s-u)^{\delta-1}}{\Gamma(\delta)} \widehat{f_{2}}(u) d u d s \\
& -\sigma_{12} \int_{0}^{\eta_{m}} \frac{\left(\eta_{m}-s\right)^{\delta-1}}{\Gamma(\delta)} \widehat{f_{2}}(s) d s+\sigma_{13} \int_{0}^{1} \frac{(1-s)^{\delta-1}}{\Gamma(\delta)} \widehat{f_{2}}(s) d s \\
& +\sigma_{14} \int_{0}^{1} \frac{(1-s)^{\delta-2}}{\Gamma(\delta-1)} \widehat{f_{2}}(s) d s-\sigma_{15} \int_{0}^{\zeta} \int_{0}^{s} \frac{(s-u)^{q-1}}{\Gamma(q)} \widehat{f_{1}}(u) d u d s \\
& \left.-\sigma_{16} \int_{0}^{\eta_{m}} \frac{\left(\eta_{m}-s\right)^{q-1}}{\Gamma(q)} \widehat{f_{1}}(s) d s+\Lambda_{3}\right]
\end{aligned}
$$

where

$$
\begin{aligned}
\Lambda_{1}= & \left(\alpha_{1}+\beta_{1}\right)\left(\alpha_{2}+\beta_{2}\right)-\left(\gamma_{1} \frac{\zeta^{2}}{2}+\sum_{m=1}^{\omega} \mu_{m} \eta_{m}\right)\left(\gamma_{2} \frac{\zeta^{2}}{2}+\sum_{m=1}^{\omega} \xi_{m} \eta_{m}\right), \\
\Lambda_{2}= & \left(\alpha_{2}+\beta_{2}\right)\left[a_{1} \alpha_{1}-b_{1} \gamma_{1} \zeta-b_{1} \sum_{m=1}^{\omega} \mu_{m}\right] \\
& +\left(\gamma_{1} \frac{\zeta^{2}}{2}+\sum_{m=1}^{\omega} \mu_{m} \eta_{m}\right)\left[b_{1} \alpha_{2}-a_{1} \gamma_{2} \zeta-a_{1} \sum_{m=1}^{\omega} \xi_{m}\right],
\end{aligned}
$$




$$
\begin{aligned}
& \Lambda_{3}=\left(\alpha_{1}+\beta_{1}\right)\left[b_{1} \alpha_{2}-a_{1} \gamma_{2} \zeta-a_{1} \sum_{m=1}^{\omega} \xi_{m}\right] \\
& +\left(\gamma_{2} \frac{\zeta^{2}}{2}+\sum_{m=1}^{\omega} \xi_{m} \eta_{m}\right)\left[a_{1} \alpha_{1}-b_{1} \gamma_{1} \zeta-b_{1} \sum_{m=1}^{\omega} \mu_{m}\right] \\
& \left\{\begin{array}{l}
\sigma_{1}=\alpha_{1}\left(\alpha_{2}+\beta_{2}\right), \quad \sigma_{7}=\gamma_{2}\left(\gamma_{1} \frac{\zeta^{2}}{2}+\sum_{m=1}^{\omega} \mu_{m} \eta_{m}\right), \quad \sigma_{13}=\alpha_{2}\left(\alpha_{1}+\beta_{1}\right), \\
\sigma_{2}=\beta_{1}\left(\alpha_{2}+\beta_{2}\right), \quad \sigma_{8}=\left(\gamma_{1} \frac{\zeta^{2}}{2}+\sum_{m=1}^{\omega} \mu_{m} \eta_{m}\right) \sum_{m=1}^{\omega} \xi_{m}, \\
\sigma_{14}=\beta_{2}\left(\alpha_{1}+\beta_{1}\right), \quad \sigma_{3}=\gamma_{1}\left(\alpha_{2}+\beta_{2}\right), \quad \sigma_{9}=\alpha_{1}\left(\gamma_{2} \frac{\zeta^{2}}{2}+\sum_{m=1}^{\omega} \xi_{m} \eta_{m}\right), \\
\sigma_{15}=\gamma_{2}\left(\alpha_{1}+\beta_{1}\right), \quad \sigma_{4}=\left(\alpha_{2}+\beta_{2}\right) \sum_{m=1}^{\omega} \mu_{m}, \\
\sigma_{10}=\beta_{1}\left(\gamma_{2} \frac{\zeta^{2}}{2}+\sum_{m=1}^{\omega} \xi_{m} \eta_{m}\right), \quad \sigma_{16}=\left(\alpha_{1}+\beta_{1}\right) \sum_{m=1}^{\omega} \xi_{m}, \\
\sigma_{5}=\alpha_{2}\left(\gamma_{1} \frac{\zeta^{2}}{2}+\sum_{m=1}^{\omega} \mu_{m} \eta_{m}\right), \quad \sigma_{11}=\gamma_{1}\left(\gamma_{2} \frac{\zeta^{2}}{2}+\sum_{m=1}^{\omega} \xi_{m} \eta_{m}\right), \\
\sigma_{6}=\beta_{2}\left(\gamma_{1} \frac{\zeta^{2}}{2}+\sum_{m=1}^{\omega} \mu_{m} \eta_{m}\right), \quad \sigma_{12}=\left(\gamma_{2} \frac{\zeta^{2}}{2}+\sum_{m=1}^{\omega} \xi_{m} \eta_{m}\right) \sum_{m=1}^{\omega} \mu_{m} .
\end{array}\right.
\end{aligned}
$$

Proof Applying the integral operators $I^{q}$ and $I^{\delta}$ respectively on the first and second equations of (4) and then using (3), we get

$$
\begin{aligned}
& x(t)=\int_{0}^{t} \frac{(t-s)^{q-1}}{\Gamma(q)} \widehat{f_{1}}(s) d s-c_{0}-c_{1} t, \\
& y(t)=\int_{0}^{t} \frac{(t-s)^{\delta-1}}{\Gamma(\delta)} \widehat{f}_{2}(s) d s-d_{0}-d_{1} t,
\end{aligned}
$$

where $c_{0}, c_{1}, d_{0}, d_{1}$ are arbitrary constants. Using the conditions $x(0)=a_{1}$ and $y(0)=b_{1}$ respectively in (8) and (9), we find that $c_{0}=-a_{1}, d_{0}=-b_{1}$; consequently, we have

$$
\begin{aligned}
& x(t)=\int_{0}^{t} \frac{(t-s)^{q-1}}{\Gamma(q)} \widehat{f_{1}}(s) d s+a_{1}-c_{1} t, \\
& y(t)=\int_{0}^{t} \frac{(t-s)^{\delta-1}}{\Gamma(\delta)} \widehat{f}_{2}(s) d s+b_{1}-d_{1} t .
\end{aligned}
$$

Using (10) and (11) in the conditions $\alpha_{1} x(1)+\beta_{1} x^{\prime}(1)=\gamma_{1} \int_{0}^{\zeta} y(s) d s+\sum_{m=1}^{\omega} \mu_{m} y\left(\eta_{m}\right)$ and $\alpha_{2} y(1)+\beta_{2} y^{\prime}(1)=\gamma_{2} \int_{0}^{\zeta} x(s) d s+\sum_{m=1}^{\omega} \xi_{m} x\left(\eta_{m}\right)$, we obtain a system of equations in the unknown constants $c_{1}$ and $d_{1}$ given by

$$
\left\{\begin{array}{l}
U_{1} c_{1}-V_{1} d_{1}=A_{1}, \\
-V_{2} c_{1}+U_{2} d_{1}=A_{2},
\end{array}\right.
$$

where

$$
\begin{aligned}
& U_{1}=\left(\alpha_{1}+\beta_{1}\right), \quad U_{2}=\left(\alpha_{2}+\beta_{2}\right), \\
& V_{1}=\left(\gamma_{1} \frac{\zeta^{2}}{2}+\sum_{m=1}^{\omega} \mu_{m} \eta_{m}\right), \quad V_{2}=\left(\gamma_{2} \frac{\zeta^{2}}{2}+\sum_{m=1}^{\omega} \xi_{m} \eta_{m}\right)
\end{aligned}
$$




$$
\begin{aligned}
A_{1}= & \alpha_{1} \int_{0}^{1} \frac{(1-s)^{q-1}}{\Gamma(q)} \widehat{f_{1}}(s) d s+\beta_{1} \int_{0}^{1} \frac{(1-s)^{q-2}}{\Gamma(q-1)} \widehat{f}_{1}(s) d s \\
& -\gamma_{1} \int_{0}^{\zeta} \int_{0}^{s} \frac{(s-u)^{\delta-1}}{\Gamma(\delta)} \widehat{f_{2}}(u) d u d s \\
& -\sum_{m=1}^{\omega} \mu_{m} \int_{0}^{\eta_{m}} \frac{\left(\eta_{m}-s\right)^{\delta-1}}{\Gamma(\delta)} \widehat{f_{2}}(s) d s+a_{1} \alpha_{1}-b_{1} \gamma_{1} \zeta-b_{1} \sum_{m=1}^{\omega} \mu_{m}, \\
A_{2}= & \alpha_{2} \int_{0}^{1} \frac{(1-s)^{\delta-1}}{\Gamma(\delta)} \widehat{f_{2}}(s) d s+\beta_{2} \int_{0}^{1} \frac{(1-s)^{\delta-2}}{\Gamma(\delta-1)} \widehat{f}_{2}(s) d s \\
& -\gamma_{2} \int_{0}^{\zeta} \int_{0}^{s} \frac{(s-u)^{q-1}}{\Gamma(q)} \widehat{f_{1}}(u) d u d s \\
& -\sum_{m=1}^{\omega} \xi_{m} \int_{0}^{\eta_{m}} \frac{\left(\eta_{m}-s\right)^{q-1}}{\Gamma(q)} \widehat{f}_{1}(s) d s+b_{1} \alpha_{2}-a_{1} \gamma_{2} \zeta-a_{1} \sum_{m=1}^{\omega} \xi_{m} .
\end{aligned}
$$

Solving system (12) for $c_{1}$ and $d_{1}$, we find that

$$
c_{1}=\frac{U_{2} A_{1}+V_{1} A_{2}}{U_{1} U_{2}-V_{1} V_{2}}, \quad d_{1}=\frac{V_{2} A_{1}+U_{1} A_{2}}{U_{1} U_{2}-V_{1} V_{2}} .
$$

Substituting the values of $c_{1}$ and $d_{1}$ in (10) and (11) respectively together with notations (13) leads to solutions (5) and (6). The converse can be proved by direct computation. The proof is completed.

\section{Existence and uniqueness results}

Let $\mathcal{S}=\{x \mid x \in C([a, b], \mathbb{R})\}$ be the space equipped with the norm $\|x\|=\sup _{t \in[0,1]}|x(t)|$. Obviously, $(\mathcal{S},\|\cdot\|)$ is a Banach space and, consequently, the product space $(\mathcal{S} \times \mathcal{S},\|\cdot\|)$ is a Banach space with the norm $\|(x+y)\|=\|x\|+\|y\|$ for $(x, y) \in \mathcal{S} \times \mathcal{S}$.

In view of Lemma 2 , we define an operator $\mathcal{Q}: \mathcal{S} \times \mathcal{S} \rightarrow \mathcal{S} \times \mathcal{S}$ by

$$
\mathcal{Q}(x, y)(t):=\left(\mathcal{Q}_{1}(x, y)(t), \mathcal{Q}_{2}(x, y)(t)\right)
$$

where

$$
\begin{aligned}
& \mathcal{Q}_{1}(x, y)(t) \\
& =\int_{0}^{t} \frac{(t-s)^{q-1}}{\Gamma(q)} f_{1}(s, x(s), y(s)) d s-\sum_{i=1}^{k} \int_{0}^{t} \frac{(t-s)^{q+p_{i}-1}}{\Gamma\left(q+p_{i}\right)} g_{i}(s, x(s), y(s)) d s+a_{1} \\
& \quad-\frac{t}{\Lambda_{1}}\left[\sigma_{1} \int_{0}^{1}\left(\frac{(1-s)^{q-1}}{\Gamma(q)} f_{1}(s, x(s), y(s))-\sum_{i=1}^{k} \frac{(1-s)^{q+p_{i}-1}}{\Gamma\left(q+p_{i}\right)} g_{i}(s, x(s), y(s))\right) d s\right. \\
& \quad+\sigma_{2} \int_{0}^{1}\left(\frac{(1-s)^{q-2}}{\Gamma(q-1)} f_{1}(s, x(s), y(s))-\sum_{i=1}^{k} \frac{(1-s)^{q+p_{i}-2}}{\Gamma\left(q+p_{i}-1\right)^{2}} g_{i}(s, x(s), y(s))\right) d s \\
& \quad-\sigma_{3}\left(\int_{0}^{\zeta} \int_{0}^{s} \frac{(s-u)^{\delta-1}}{\Gamma(\delta)} f_{2}(u, x(u), y(u)) d u d s\right. \\
& \left.\quad+\sum_{j=1}^{l} \int_{0}^{\zeta} \int_{0}^{s} \frac{(s-u)^{\delta-1}}{\Gamma(\delta)} \int_{0}^{u} \frac{(u-w)^{v_{j}-1}}{\Gamma\left(v_{j}\right)} h_{j}(w, x(w), y(w)) d w d u d s\right)
\end{aligned}
$$




$$
\begin{aligned}
& -\sigma_{4} \int_{0}^{\eta_{m}}\left(\frac{\left(\eta_{m}-s\right)^{\delta-1}}{\Gamma(\delta)} f_{2}(s, x(s), y(s))+\sum_{j=1}^{l} \frac{\left(\eta_{m}-s\right)^{\delta+v_{j}-1}}{\Gamma\left(\delta+v_{j}\right)} h_{j}(s, x(s), y(s))\right) d s \\
& +\sigma_{5} \int_{0}^{1}\left(\frac{(1-s)^{\delta-1}}{\Gamma(\delta)} f_{2}(s, x(s), y(s))-\sum_{j=1}^{l} \frac{(1-s)^{\delta+v_{j}-1}}{\Gamma\left(\delta+v_{j}\right)} h_{j}(s, x(s), y(s))\right) d s \\
& +\sigma_{6} \int_{0}^{1}\left(\frac{(1-s)^{\delta-2}}{\Gamma(\delta-1)} f_{2}(s, x(s), y(s))-\sum_{j=1}^{l} \frac{(1-s)^{\delta+v_{j}-2}}{\Gamma\left(\delta+v_{j}-1\right)} h_{j}(s, x(s), y(s))\right) d s \\
& -\sigma_{7}\left(\int_{0}^{\zeta} \int_{0}^{s} \frac{(s-u)^{q-1}}{\Gamma(q)} f_{1}(u, x(u), y(u)) d u d s\right. \\
& \left.+\sum_{i=1}^{k} \int_{0}^{\zeta} \int_{0}^{s} \frac{(s-u)^{q-1}}{\Gamma(q)} \int_{0}^{u} \frac{(u-w)^{p_{i}-1}}{\Gamma\left(p_{i}\right)} g_{i}(w, x(w), y(w)) d w d u d s\right) \\
& -\sigma_{8} \int_{0}^{\eta_{m}}\left(\frac{\left(\eta_{m}-s\right)^{q-1}}{\Gamma(q)} f_{1}(s, x(s), y(s))\right. \\
& \left.\left.+\sum_{i=1}^{k} \frac{\left(\eta_{m}-s\right)^{q+p_{i}-1}}{\Gamma\left(q+p_{i}\right)} g_{i}(s, x(s), y(s))\right) d s+\Lambda_{2}\right]
\end{aligned}
$$

and

$$
\begin{aligned}
& \mathcal{Q}_{2}(x, y)(t) \\
& =\int_{0}^{t} \frac{(t-s)^{\delta-1}}{\Gamma(\delta)} f_{2}(s, x(s), y(s)) d s-\sum_{j=1}^{l} \int_{0}^{t} \frac{(t-s)^{\delta+v_{j}-1}}{\Gamma\left(\delta+v_{j}\right)} h_{j}(s, x(s), y(s)) d s+b_{1} \\
& -\frac{t}{\Lambda_{1}}\left[\sigma _ { 9 } \int _ { 0 } ^ { 1 } \left(\frac{(1-s)^{q-1}}{\Gamma(q)} f_{1}(s, x(s), y(s))-\sum_{i=1}^{k} \frac{(1-s)^{q+p_{i}-1}}{\Gamma\left(q+p_{i}\right)} g_{i}(s, x(s), y(s)) d s\right.\right. \\
& +\sigma_{10} \int_{0}^{1}\left(\frac{(1-s)^{q-2}}{\Gamma(q-1)} f_{1}(s, x(s), y(s))-\sum_{i=1}^{k} \frac{(1-s)^{q+p_{i}-2}}{\Gamma\left(q+p_{i}-1\right)} g_{i}(s, x(s), y(s))\right) d s \\
& -\sigma_{11}\left(\int_{0}^{\zeta} \int_{0}^{s} \frac{(s-u)^{\delta-1}}{\Gamma(\delta)} f_{2}(u, x(u), y(u)) d u d s\right. \\
& \left.+\sum_{j=1}^{l} \int_{0}^{\zeta} \int_{0}^{s} \frac{(s-u)^{\delta-1}}{\Gamma(\delta)} \int_{0}^{u} \frac{(u-w)^{v_{j}-1}}{\Gamma\left(v_{j}\right)} h_{j}(w, x(w), y(w)) d w d u d s\right) \\
& -\sigma_{12} \int_{0}^{\eta_{m}}\left(\frac{\left(\eta_{m}-s\right)^{\delta-1}}{\Gamma(\delta)} f_{2}(s, x(s), y(s))+\sum_{j=1}^{l} \frac{\left(\eta_{m}-s\right)^{\delta+v_{j}-1}}{\Gamma\left(\delta+v_{j}\right)} h_{j}(s, x(s), y(s))\right) d s \\
& +\sigma_{13} \int_{0}^{1}\left(\frac{(1-s)^{\delta-1}}{\Gamma(\delta)} f_{2}(s, x(s), y(s))-\sum_{j=1}^{l} \frac{(1-s)^{\delta+v_{j}-1}}{\Gamma\left(\delta+v_{j}\right)} h_{j}(s, x(s), y(s))\right) d s \\
& +\sigma_{14} \int_{0}^{1}\left(\frac{(1-s)^{\delta-2}}{\Gamma(\delta-1)} f_{2}(s, x(s), y(s))-\sum_{j=1}^{l} \frac{(1-s)^{\delta+v_{j}-2}}{\Gamma\left(\delta+v_{j}-1\right)} h_{j}(s, x(s), y(s))\right) d s \\
& -\sigma_{15}\left(\int_{0}^{\zeta} \int_{0}^{s} \frac{(s-u)^{q-1}}{\Gamma(q)} f_{1}(u, x(u), y(u)) d u d s\right.
\end{aligned}
$$




$$
\begin{aligned}
& \left.+\sum_{i=1}^{k} \int_{0}^{\zeta} \int_{0}^{s} \frac{(s-u)^{q-1}}{\Gamma(q)} \int_{0}^{u} \frac{(u-w)^{p_{i}-1}}{\Gamma\left(p_{i}\right)} g_{i}(w, x(w), y(w)) d w d u d s\right) \\
& -\sigma_{16} \int_{0}^{\eta_{m}}\left(\frac{\left(\eta_{m}-s\right)^{q-1}}{\Gamma(q)} f_{1}(s, x(s), y(s))\right. \\
& \left.\left.+\sum_{i=1}^{k} \frac{\left(\eta_{m}-s\right)^{q+p_{i}-1}}{\Gamma\left(q+p_{i}\right)} g_{i}(s, x(s), y(s))\right) d s+\Lambda_{3}\right] .
\end{aligned}
$$

In the sequel, we use the following notations:

$$
\begin{aligned}
& \varphi_{1}=\left[\frac{1}{\Gamma(q+1)}+\frac{1}{\left|\Lambda_{1}\right|}\left(\frac{\left|\sigma_{1}\right|+\left|\sigma_{8}\right| \eta_{m}^{q}}{\Gamma(q+1)}+\frac{\left|\sigma_{2}\right|}{\Gamma(q)}+\frac{\left|\sigma_{7}\right| \zeta^{q+1}}{\Gamma(q+2)}\right)\right], \\
& \varphi_{2}=\left[\frac{1}{\left|\Lambda_{1}\right|}\left(\frac{\left|\sigma_{3}\right| \zeta^{\delta+1}}{\Gamma(\delta+2)}+\frac{\left|\sigma_{4}\right| \eta_{m}^{\delta}+\left|\sigma_{5}\right|}{\Gamma(\delta+1)}+\frac{\left|\sigma_{6}\right|}{\Gamma(\delta)}\right)\right], \\
& \Omega_{i}=\left[\frac{1}{\Gamma\left(q+p_{i}+1\right)}+\frac{1}{\left|\Lambda_{1}\right|}\left(\frac{\left|\sigma_{1}\right|+\left|\sigma_{8}\right| \eta_{m}^{q+p_{i}}}{\Gamma\left(q+p_{i}+1\right)}+\frac{\left|\sigma_{2}\right|}{\Gamma\left(q+p_{i}\right)}+\frac{\left|\sigma_{7}\right| \zeta^{q+p_{i}+1}}{\Gamma\left(q+p_{i}+2\right)}\right)\right], \\
& \widehat{\Omega}_{j}=\left[\frac{1}{\left|\Lambda_{1}\right|}\left(\frac{\left|\sigma_{3}\right| \zeta^{\delta+v_{j}+1}}{\Gamma\left(\delta+v_{j}+2\right)}+\frac{\left|\sigma_{4}\right| \eta_{m}^{\delta+v_{j}}+\left|\sigma_{5}\right|}{\Gamma\left(\delta+v_{j}+1\right)}+\frac{\left|\sigma_{6}\right|}{\Gamma\left(\delta+v_{j}\right)}\right)\right], \\
& \vartheta_{1}=\left[\frac{1}{\left|\Lambda_{1}\right|}\left(\frac{\left|\sigma_{9}\right|+\left|\sigma_{16}\right| \eta_{m}^{q}}{\Gamma(q+1)}+\frac{\left|\sigma_{10}\right|}{\Gamma(q)}+\frac{\left|\sigma_{15}\right| \zeta^{q+1}}{\Gamma(q+2)}\right)\right], \\
& \vartheta_{2}=\left[\frac{1}{\Gamma(\delta+1)}+\frac{1}{\left|\Lambda_{1}\right|}\left(\frac{\left|\sigma_{11}\right| \zeta^{\delta+1}}{\Gamma(\delta+2)}+\frac{\left|\sigma_{12}\right| \eta_{m}^{\delta}+\left|\sigma_{13}\right|}{\Gamma(\delta+1)}+\frac{\left|\sigma_{14}\right|}{\Gamma(\delta)}\right)\right], \\
& \Theta_{i}=\left[\frac{1}{\left|\Lambda_{1}\right|}\left(\frac{\left|\sigma_{9}\right|+\left|\sigma_{16}\right| \eta_{m}^{q+p_{i}}}{\Gamma\left(q+p_{i}+1\right)}+\frac{\left|\sigma_{10}\right|}{\Gamma\left(q+p_{i}\right)}+\frac{\left|\sigma_{15}\right| \zeta^{q+p_{i}+1}}{\Gamma\left(q+p_{i}+2\right)}\right)\right], \\
& \widehat{\Theta}_{j}=\left[\frac{1}{\Gamma\left(\delta+v_{j}+1\right)}+\frac{1}{\left|\Lambda_{1}\right|}\left(\frac{\left|\sigma_{11}\right| \zeta^{\delta+v_{j}+1}}{\Gamma\left(\delta+v_{j}+2\right)}+\frac{\left|\sigma_{12}\right| \eta_{m}^{\delta+v_{j}}+\left|\sigma_{13}\right|}{\Gamma\left(\delta+v_{j}+1\right)}+\frac{\left|\sigma_{14}\right|}{\Gamma\left(\delta+v_{j}\right)}\right)\right] .
\end{aligned}
$$

Now we prove the existence and uniqueness of solutions for system (1) by applying the Banach contraction mapping principle.

Theorem 1 Let $f_{1}, f_{2}, g_{i}, h_{j}:[0,1] \times \mathbb{R} \times \mathbb{R} \rightarrow \mathbb{R}(i=1, \ldots, k)(j=1, \ldots, l)$ be continuous functions. In addition, we assume that:

$\left(H_{1}\right)$ There exist constants $L_{1}, L_{2}>0$ such that, $\forall t \in[0,1]$ and $x_{\epsilon}, y_{\epsilon} \in \mathbb{R}, \epsilon=1,2$,

$$
\begin{aligned}
& \left|f_{1}\left(t, x_{1}, y_{1}\right)-f_{1}\left(t, x_{2}, y_{2}\right)\right| \leq L_{1}\left(\left|x_{1}-y_{1}\right|+\left|x_{2}-y_{2}\right|\right), \\
& \left|f_{2}\left(t, x_{1}, y_{1}\right)-f_{2}\left(t, x_{2}, y_{2}\right)\right| \leq L_{2}\left(\left|x_{1}-y_{1}\right|+\left|x_{2}-y_{2}\right|\right) .
\end{aligned}
$$

$\left(H_{2}\right)$ There exist constants $M_{i}, \widehat{M}_{j}>0(i=1, \ldots, k)(j=1, \ldots, l)$ such that $\forall t \in[0,1]$ and $x_{\epsilon}, y_{\epsilon} \in \mathbb{R}, \epsilon=1,2$,

$$
\begin{aligned}
& \left|g_{i}\left(t, x_{1}, y_{1}\right)-g_{i}\left(t, x_{2}, y_{2}\right)\right| \leq M_{i}\left(\left|x_{1}-y_{1}\right|+\left|x_{2}-y_{2}\right|\right), \\
& \left|h_{j}\left(t, x_{1}, y_{1}\right)-h_{j}\left(t, x_{2}, y_{2}\right)\right| \leq \widehat{M}_{j}\left(\left|x_{1}-y_{1}\right|+\left|x_{2}-y_{2}\right|\right) .
\end{aligned}
$$


Then system (1)-(2) has a unique solution on $[0,1]$, provided that $\Psi<1$, where

$$
\Psi=L_{1}\left(\varphi_{1}+\vartheta_{1}\right)+L_{2}\left(\varphi_{2}+\vartheta_{2}\right)+\sum_{i=1}^{k} M_{i}\left(\Omega_{i}+\Theta_{i}\right)+\sum_{j=1}^{l} \widehat{M}_{j}\left(\widehat{\Omega}_{j}+\widehat{\Theta}_{j}\right)
$$

Proof Let us define finite numbers $W_{1}, W_{2}, N_{i}, \widehat{N}_{j}$ as follows:

$$
\begin{array}{ll}
W_{1}=\sup _{t \in[0,1]}\left|f_{1}(t, 0,0)\right|, & W_{2}=\sup _{t \in[0,1]}\left|f_{2}(t, 0,0)\right|, \\
N_{i}=\sup _{t \in[0,1]}\left|g_{i}(t, 0,0)\right|, & \widehat{N}_{j}=\sup _{t \in[0,1]}\left|h_{j}(t, 0,0)\right|,
\end{array}
$$

and show that $\mathcal{Q} B_{r} \subset B_{r}$, where $B_{r}=\{(x, y) \in \mathcal{S} \times \mathcal{S}:\|(x, y)\| \leq r\}$ with

$$
r>\frac{W_{1}\left(\varphi_{1}+\vartheta_{1}\right)+W_{2}\left(\varphi_{2}+\vartheta_{2}\right)+\sum_{i=1}^{k} N_{i}\left(\Omega+\Theta_{i}\right)+\sum_{j=1}^{l} \widehat{N}_{j}\left(\widehat{\Omega}_{j}+\widehat{\Theta}_{j}\right)+\left|a_{1}\right|+\left|b_{1}\right|+\left(\left|\Lambda_{2}+\Lambda_{3}\right|\right) /\left|\Lambda_{1}\right|}{1-\Psi} .
$$

For any $(x, y) \in B_{r}, t \in[0,1]$, using $\left(H_{1}\right)$, we get

$$
\begin{aligned}
\left|f_{1}(t, x, y)\right| & =\left|f_{1}(t, x, y)-f_{1}(t, 0,0)+f_{1}(t, 0,0)\right| \\
& \leq\left|f_{1}(t, x, y)-f_{1}(t, 0,0)\right|+\left|f_{1}(t, 0,0)\right| \\
& \leq L_{1}(|x(t)|+|y(t)|)+W_{1} \leq L_{1}(\|x\|+\|y\|)+W_{1} \leq L_{1} r+W_{1} .
\end{aligned}
$$

Similarly, we can find that

$$
\left|f_{2}(t, x, y)\right| \leq L_{2} r+W_{2}, \quad\left|g_{i}(t, x, y)\right| \leq M_{i} r+N_{i}, \quad\left|h_{j}(t, x, y)\right| \leq \widehat{M}_{j} r+\widehat{N}_{j}
$$

Then

$$
\begin{aligned}
& \left\|\mathcal{Q}_{1}(x, y)\right\| \\
& \leq \sup _{t \in[0,1]}\left\{\int_{0}^{t} \frac{(t-s)^{q-1}}{\Gamma(q)}\left|f_{1}(s, x(s), y(s))\right| d s+\sum_{i=1}^{k} \int_{0}^{t} \frac{(t-s)^{q+p_{i}-1}}{\Gamma\left(q+p_{i}\right)}\left|g_{i}(s, x(s), y(s))\right| d s\right. \\
& \quad+\left|a_{1}\right|+\frac{t}{\left|\Lambda_{1}\right|}\left[| \sigma _ { 1 } | \int _ { 0 } ^ { 1 } \left(\frac{(1-s)^{q-1}}{\Gamma(q)}\left|f_{1}(s, x(s), y(s))\right|\right.\right. \\
& \left.\quad+\sum_{i=1}^{k} \frac{(1-s)^{q+p_{i}-1}}{\Gamma\left(q+p_{i}\right)}\left|g_{i}(s, x(s), y(s))\right|\right) d s \\
& \quad+\left|\sigma_{2}\right| \int_{0}^{1}\left(\frac{(1-s)^{q-2}}{\Gamma(q-1)}\left|f_{1}(s, x(s), y(s))\right|+\sum_{i=1}^{k} \frac{(1-s)^{q+p_{i}-2}}{\Gamma\left(q+p_{i}-1\right)}\left|g_{i}(s, x(s), y(s))\right|\right) d s \\
& \quad+\left|\sigma_{3}\right|\left(\int_{0}^{\zeta} \int_{0}^{s} \frac{(s-u)^{\delta-1}}{\Gamma(\delta)}\left|f_{2}(u, x(u), y(u))\right| d u d s\right. \\
& \left.\quad+\sum_{j=1}^{l} \int_{0}^{\zeta} \int_{0}^{s} \frac{(s-u)^{\delta-1}}{\Gamma(\delta)} \int_{0}^{u} \frac{(u-w)^{v_{j}-1}}{\Gamma\left(v_{j}\right)}\left|h_{j}(w, x(w), y(w))\right| d w d u d s\right)
\end{aligned}
$$




$$
\begin{aligned}
& +\left|\sigma_{4}\right| \int_{0}^{\eta_{m}}\left(\frac{\left(\eta_{m}-s\right)^{\delta-1}}{\Gamma(\delta)}\left|f_{2}(s, x(s), y(s))\right|+\sum_{j=1}^{l} \frac{\left(\eta_{m}-s\right)^{\delta+v_{j}-1}}{\Gamma\left(\delta+v_{j}\right)}\left|h_{j}(s, x(s), y(s))\right|\right) d s \\
& +\left|\sigma_{5}\right| \int_{0}^{1}\left(\frac{(1-s)^{\delta-1}}{\Gamma(\delta)}\left|f_{2}(s, x(s), y(s))\right|+\sum_{j=1}^{l} \frac{(1-s)^{\delta+v_{j}-1}}{\Gamma\left(\delta+v_{j}\right)}\left|h_{j}(s, x(s), y(s))\right|\right) d s \\
& +\left|\sigma_{6}\right| \int_{0}^{1}\left(\frac{(1-s)^{\delta-2}}{\Gamma(\delta-1)}\left|f_{2}(s, x(s), y(s))\right|+\sum_{j=1}^{l} \frac{(1-s)^{\delta+v_{j}-2}}{\Gamma\left(\delta+v_{j}-1\right)}\left|h_{j}(s, x(s), y(s))\right|\right) d s \\
& +\left|\sigma_{7}\right|\left(\int_{0}^{\zeta} \int_{0}^{s} \frac{(s-u)^{q-1}}{\Gamma(q)}\left|f_{1}(u, x(u), y(u))\right| d u d s\right. \\
& \left.+\sum_{i=1}^{k} \int_{0}^{\zeta} \int_{0}^{s} \frac{(s-u)^{q-1}}{\Gamma(q)} \int_{0}^{u} \frac{(u-w)^{p_{i}-1}}{\Gamma\left(p_{i}\right)}\left|g_{i}(w, x(w), y(w))\right| d w d u d s\right) \\
& +\left|\sigma_{8}\right| \int_{0}^{\eta_{m}}\left(\frac{\left(\eta_{m}-s\right)^{q-1}}{\Gamma(q)}\left|f_{1}(s, x(s), y(s))\right|\right. \\
& \left.\left.\left.+\sum_{i=1}^{k} \frac{\left(\eta_{m}-s\right)^{q+p_{i}-1}}{\Gamma\left(q+p_{i}\right)}\left|g_{i}(s, x(s), y(s))\right|\right) d s+\left|\Lambda_{2}\right|\right]\right\} \\
& \leq\left(L_{1} r+W_{1}\right) \sup _{t \in[0,1]}\left\{\left[\frac{t^{q}}{\Gamma(q+1)}+\frac{t}{\left|\Lambda_{1}\right|}\left(\frac{\left|\sigma_{1}\right|}{\Gamma(q+1)}+\frac{\left|\sigma_{2}\right|}{\Gamma(q)}+\frac{\left|\sigma_{7}\right| \zeta^{q+1}}{\Gamma(q+2)}+\frac{\left|\sigma_{8}\right| \eta_{m}^{q}}{\Gamma(q+1)}\right)\right]\right. \\
& +\left(L_{2} r+W_{2}\right)\left[\frac{t}{\left|\Lambda_{1}\right|}\left(\frac{\left|\sigma_{3}\right| \zeta^{\delta+1}}{\Gamma(\delta+2)}+\frac{\left|\sigma_{4}\right| \eta_{m}^{\delta}}{\Gamma(\delta+1)}+\frac{\left|\sigma_{5}\right|}{\Gamma(\delta+1)}+\frac{\left|\sigma_{6}\right|}{\Gamma(\delta)}\right)\right] \\
& +\sum_{i=1}^{k}\left(M_{i} r+N_{i}\right)\left[\frac{t^{q+p_{i}}}{\Gamma\left(q+p_{i}+1\right)}+\frac{t}{\left|\Lambda_{1}\right|}\left(\frac{\left|\sigma_{1}\right|}{\Gamma\left(q+p_{i}+1\right)}+\frac{\left|\sigma_{2}\right|}{\Gamma\left(q+p_{i}\right)}+\frac{\left|\sigma_{7}\right| \zeta^{q+p_{i}+1}}{\Gamma\left(q+p_{i}+2\right)}\right.\right. \\
& \left.\left.+\frac{\left|\sigma_{8}\right| \eta_{m}^{q+p_{i}}}{\Gamma\left(q+p_{i}+1\right)}\right)\right]+\sum_{j=1}^{l}\left(\widehat{M}_{j} r+\widehat{N}_{j}\right)\left[\frac { t } { | \Lambda _ { 1 } | } \left(\frac{\left|\sigma_{3}\right| \zeta^{\delta+v_{j}+1}}{\Gamma\left(\delta+v_{j}+2\right)}+\frac{\left|\sigma_{4}\right| \eta_{m}^{\delta+v_{j}}}{\Gamma\left(\delta+v_{j}+1\right)}\right.\right. \\
& \left.\left.\left.+\frac{\left|\sigma_{5}\right|}{\Gamma\left(\delta+v_{j}+1\right)}+\frac{\left|\sigma_{6}\right|}{\Gamma\left(\delta+v_{j}\right)}\right)\right]+\left(\left|a_{1}\right|+\frac{t\left|\Lambda_{2}\right|}{\left|\Lambda_{1}\right|}\right)\right\} \\
& \leq\left(L_{1} \varphi_{1}+L_{2} \varphi_{2}+\sum_{i=1}^{k} M_{i} \Omega_{i}+\sum_{j=1}^{l} \widehat{M}_{j} \widehat{\Omega}_{j}\right) r+W_{1} \varphi_{1}+W_{2} \varphi_{2}+\sum_{i=1}^{k} N_{i} \Omega_{i} \\
& +\sum_{j=1}^{l} \widehat{N}_{j} \widehat{\Omega}_{j}+\left|a_{1}\right|+\left|\frac{\Lambda_{2}}{\Lambda_{1}}\right| .
\end{aligned}
$$

Similarly, we can find that

$$
\begin{aligned}
\left\|\mathcal{Q}_{2}(x, y)\right\| \leq & \left(L_{1} \vartheta_{1}+L_{2} \vartheta_{2}+\sum_{i=1}^{k} M_{i} \Theta_{i}+\sum_{j=1}^{l} \widehat{M}_{j} \widehat{\Theta}_{j}\right) r+W_{1} \vartheta_{1}+W_{2} \vartheta_{2}+\sum_{i=1}^{k} N_{i} \Theta_{i} \\
& +\sum_{j=1}^{l} \widehat{N}_{j} \widehat{\Theta}_{j}+\left|b_{1}\right|+\left|\frac{\Lambda_{3}}{\Lambda_{1}}\right| .
\end{aligned}
$$


Consequently, in view of (16), we get

$$
\begin{aligned}
\|\mathcal{Q}(x, y)\| \leq & \Psi r+W_{1}\left(\varphi_{1}+\vartheta_{1}\right)+W_{2}\left(\varphi_{2}+\vartheta_{2}\right)+\sum_{i=1}^{k} N_{i}\left(\Omega_{i}+\Theta_{i}\right)+\sum_{j=1}^{l} \widehat{N}_{j}\left(\widehat{\Omega}_{j}+\widehat{\Theta}_{j}\right) \\
& +\left|a_{1}\right|+\left|b_{1}\right|+\left|\frac{\Lambda_{2}+\Lambda_{3}}{\Lambda_{1}}\right| \leq r
\end{aligned}
$$

which implies that $\mathcal{Q} B_{r} \subset B_{r}$. Next we show that the operator $\mathcal{Q}$ is a contraction. Using conditions $\left(H_{1}\right)$ and $\left(H_{2}\right)$, for any $\left(x_{1}, y_{1}\right),\left(x_{2}, y_{2}\right) \in \mathcal{S} \times \mathcal{S}, t \in[0,1]$, we get

$$
\begin{aligned}
& \left\|\mathcal{Q}_{1}\left(x_{1}, y_{1}\right)-\mathcal{Q}_{1}\left(x_{2}, y_{2}\right)\right\| \\
& \leq \sup _{t \in[0,1]}\left\{\int_{0}^{t} \frac{(t-s)^{q-1}}{\Gamma(q)}\left|f_{1}\left(s, x_{1}(s), y_{1}(s)\right)-f_{1}\left(s, x_{2}(s), y_{2}(s)\right)\right| d s\right. \\
& +\sum_{i=1}^{k} \int_{0}^{t} \frac{(t-s)^{q+p_{i}-1}}{\Gamma\left(q+p_{i}\right)}\left|g_{i}\left(s, x_{1}(s), y_{1}(s)\right)-g_{i}\left(s, x_{2}(s), y_{2}(s)\right)\right| d s \\
& +\frac{t}{\left|\Lambda_{1}\right|}\left[| \sigma _ { 1 } | \int _ { 0 } ^ { 1 } \left(\frac{(1-s)^{q-1}}{\Gamma(q)}\left|f_{1}\left(s, x_{1}(s), y_{1}(s)\right)-f_{1}\left(s, x_{2}(s), y_{2}(s)\right)\right|\right.\right. \\
& \left.+\sum_{i=1}^{k} \frac{(1-s)^{q+p_{i}-1}}{\Gamma\left(q+p_{i}\right)}\left|g_{i}\left(s, x_{1}(s), y_{1}(s)\right)-g_{i}\left(s, x_{2}(s), y_{2}(s)\right)\right|\right) d s \\
& +\left|\sigma_{2}\right| \int_{0}^{1}\left(\frac{(1-s)^{q-2}}{\Gamma(q-1)}\left|f_{1}\left(s, x_{1}(s), y_{1}(s)\right)-f_{1}\left(s, x_{2}(s), y_{2}(s)\right)\right|\right. \\
& \left.+\sum_{i=1}^{k} \frac{(1-s)^{q+p_{i}-2}}{\Gamma\left(q+p_{i}-1\right)}\left|g_{i}\left(s, x_{1}(s), y_{1}(s)\right)-g_{i}\left(s, x_{2}(s), y_{2}(s)\right)\right|\right) d s \\
& +\left|\sigma_{3}\right|\left(\int_{0}^{\zeta} \int_{0}^{s} \frac{(s-u)^{\delta-1}}{\Gamma(\delta)}\left|f_{2}\left(u, x_{1}(u), y_{1}(u)\right)-f_{2}\left(u, x_{2}(u), y_{2}(u)\right)\right| d u d s\right. \\
& +\sum_{j=1}^{l} \int_{0}^{\zeta} \int_{0}^{s} \frac{(s-u)^{\delta-1}}{\Gamma(\delta)} \int_{0}^{u} \frac{(u-w)^{v_{j}-1}}{\Gamma\left(v_{j}\right)} \mid h_{j}\left(w, x_{1}(w), y_{1}(w)\right) \\
& \left.-h_{j}\left(w, x_{2}(w), y_{2}(w)\right) \mid d w d u d s\right) \\
& +\left|\sigma_{4}\right| \int_{0}^{\eta_{m}}\left(\frac{\left(\eta_{m}-s\right)^{\delta-1}}{\Gamma(\delta)}\left|f_{2}\left(s, x_{1}(s), y_{1}(s)\right)-f_{2}\left(s, x_{2}(s), y_{2}(s)\right)\right|\right. \\
& \left.+\sum_{j=1}^{l} \frac{\left(\eta_{m}-s\right)^{\delta+v_{j}-1}}{\Gamma\left(\delta+v_{j}\right)}\left|h_{j}\left(s, x_{1}(s), y_{1}(s)\right)-h_{j}\left(s, x_{2}(s), y_{2}(s)\right)\right|\right) d s \\
& +\left|\sigma_{5}\right| \int_{0}^{1}\left(\frac{(1-s)^{\delta-1}}{\Gamma(\delta)}\left|f_{2}\left(s, x_{1}(s), y_{1}(s)\right)-f_{2}\left(s, x_{2}(s), y_{2}(s)\right)\right|\right.
\end{aligned}
$$




$$
\begin{aligned}
& \left.+\sum_{j=1}^{l} \frac{(1-s)^{\delta+v_{j}-1}}{\Gamma\left(\delta+v_{j}\right)}\left|h_{j}\left(s, x_{1}(s), y_{1}(s)\right)-h_{j}\left(s, x_{2}(s), y_{2}(s)\right)\right|\right) d s \\
& +\left|\sigma_{6}\right| \int_{0}^{1}\left(\frac{(1-s)^{\delta-2}}{\Gamma(\delta-1)}\left|f_{2}\left(s, x_{1}(s), y_{1}(s)\right)-f_{2}\left(s, x_{2}(s), y_{2}(s)\right)\right|\right. \\
& \left.+\sum_{j=1}^{l} \frac{(1-s)^{\delta+v_{j}-2}}{\Gamma\left(\delta+v_{j}-1\right)}\left|h_{j}\left(s, x_{1}(s), y_{1}(s)\right)-h_{j}\left(s, x_{2}(s), y_{2}(s)\right)\right|\right) d s \\
& +\left|\sigma_{7}\right|\left(\int_{0}^{\zeta} \int_{0}^{s} \frac{(s-u)^{q-1}}{\Gamma(q)}\left|f_{1}\left(u, x_{1}(u), y_{1}(u)\right)-f_{1}\left(u, x_{2}(u), y_{2}(u)\right)\right| d u d s\right. \\
& +\sum_{i=1}^{k} \int_{0}^{\zeta} \int_{0}^{s} \frac{(s-u)^{q-1}}{\Gamma(q)} \int_{0}^{u} \frac{(u-w)^{p_{i}-1}}{\Gamma\left(p_{i}\right)} \mid g_{i}\left(w, x_{1}(w), y_{1}(w)\right) \\
& \left.-g_{i}\left(w, x_{2}(w), y_{2}(w)\right) \mid d w d u d s\right) \\
& +\left|\sigma_{8}\right| \int_{0}^{\eta_{m}}\left(\frac{\left(\eta_{m}-s\right)^{q-1}}{\Gamma(q)}\left|f_{1}\left(s, x_{1}(s), y_{1}(s)\right)-f_{1}\left(s, x_{2}(s), y_{2}(s)\right)\right|\right. \\
& \left.\left.\left.+\sum_{i=1}^{k} \frac{\left(\eta_{m}-s\right)^{q+p_{i}-1}}{\Gamma\left(q+p_{i}\right)}\left|g_{i}\left(s, x_{1}(s), y_{1}(s)\right)-g_{i}\left(s, x_{2}(s), y_{2}(s)\right)\right|\right) d s\right]\right\} \\
& \leq L_{1}\left(\left\|x_{1}-x_{2}\right\|+\left\|y_{1}-y_{2}\right\|\right) \sup _{t \in[0,1]}\left\{\left[\frac{t^{q}}{\Gamma(q+1)}+\frac{t}{\left|\Lambda_{1}\right|}\left(\frac{\left|\sigma_{1}\right|}{\Gamma(q+1)}+\frac{\left|\sigma_{2}\right|}{\Gamma(q)}+\frac{\left|\sigma_{7}\right| \zeta^{q+1}}{\Gamma(q+2)}\right.\right.\right. \\
& \left.\left.+\frac{\left|\sigma_{8}\right| \eta_{m}^{q}}{\Gamma(q+1)}\right)\right]+L_{2}\left(\left\|x_{1}-x_{2}\right\|+\left\|y_{1}-y_{2}\right\|\right) \\
& \times\left[\frac{t}{\left|\Lambda_{1}\right|}\left(\frac{\left|\sigma_{3}\right| \zeta^{\delta+1}}{\Gamma(\delta+2)}+\frac{\left|\sigma_{4}\right| \eta_{m}^{\delta}}{\Gamma(\delta+1)}+\frac{\left|\sigma_{5}\right|}{\Gamma(\delta+1)}+\frac{\left|\sigma_{6}\right|}{\Gamma(\delta)}\right)\right] \\
& +\sum_{i=1}^{k} M_{i}\left(\left\|x_{1}-x_{2}\right\|+\left\|y_{1}-y_{2}\right\|\right)\left[\frac{t^{q+p_{i}}}{\Gamma\left(q+p_{i}+1\right)}+\frac{t}{\left|\Lambda_{1}\right|}\left(\frac{\left|\sigma_{1}\right|}{\Gamma\left(q+p_{i}+1\right)}+\frac{\left|\sigma_{2}\right|}{\Gamma\left(q+p_{i}\right)}\right.\right. \\
& \left.\left.+\frac{\left|\sigma_{7}\right| \zeta^{q+p_{i}+1}}{\Gamma\left(q+p_{i}+2\right)}+\frac{\left|\sigma_{8}\right| \eta_{m}^{q+p_{i}}}{\Gamma\left(q+p_{i}+1\right)}\right)\right]+\sum_{j=1}^{l} \widehat{M}_{j}\left(\left\|x_{1}-x_{2}\right\|+\left\|y_{1}-y_{2}\right\|\right) \\
& \left.\times\left[\frac{t}{\left|\Lambda_{1}\right|}\left(\frac{\left|\sigma_{3}\right| \zeta^{\delta+v_{j}+1}}{\Gamma\left(\delta+v_{j}+2\right)}+\frac{\left|\sigma_{4}\right| \eta_{m}^{\delta+v_{j}}}{\Gamma\left(\delta+v_{j}+1\right)}+\frac{\left|\sigma_{5}\right|}{\Gamma\left(\delta+v_{j}+1\right)}+\frac{\left|\sigma_{6}\right|}{\Gamma\left(\delta+v_{j}\right)}\right)\right]\right\} \\
& \leq\left(L_{1} \varphi_{1}+L_{2} \varphi_{2}+\sum_{i=1}^{k} M_{i} \Omega_{i}+\sum_{j=1}^{l} \widehat{M}_{j} \widehat{\Omega}_{j}\right)\left(\left\|x_{1}-x_{2}\right\|+\left\|y_{1}-y_{2}\right\|\right),
\end{aligned}
$$

which yields

$$
\begin{aligned}
& \left\|\mathcal{Q}_{1}\left(x_{1}, y_{1}\right)-\mathcal{Q}_{1}\left(x_{2}, y_{2}\right)\right\| \\
& \quad \leq\left(L_{1} \varphi_{1}+L_{2} \varphi_{2}+\sum_{i=1}^{k} M_{i} \Omega_{i}+\sum_{j=1}^{l} \widehat{M}_{j} \widehat{\Omega}_{j}\right)\left(\left\|x_{1}-x_{2}\right\|+\left\|y_{1}-y_{2}\right\|\right) .
\end{aligned}
$$


Similarly, we find that

$$
\begin{aligned}
& \left\|\mathcal{Q}_{2}\left(x_{1}, y_{1}\right)-\mathcal{Q}_{2}\left(x_{2}, y_{2}\right)\right\| \\
& \quad \leq\left(L_{1} \vartheta_{1}+L_{2} \vartheta_{2}+\sum_{i=1}^{k} M_{i} \Theta_{i}+\sum_{j=1}^{l} \widehat{M}_{j} \widehat{\Theta}_{j}\right)\left(\left\|x_{1}-x_{2}\right\|+\left\|y_{1}-y_{2}\right\|\right) .
\end{aligned}
$$

Consequently, we get

$$
\left\|\mathcal{Q}\left(x_{1}, y_{1}\right)-\mathcal{Q}\left(x_{2}, y_{2}\right)\right\| \leq \Psi\left(\left\|x_{1}-x_{2}\right\|+\left\|y_{1}-y_{2}\right\|\right)
$$

which shows that $\mathcal{Q}$ is a contraction in view of the given condition $\Psi<1$. So, by the Banach contraction mapping principle, the operator $\mathcal{Q}$ has a unique fixed point. Therefore, system (1)-(2) has a unique solution on $[0,1]$. This completes the proof.

In the following result, we apply the Leray-Schauder alternative [23] to prove the existence of solutions for system (1)-(2).

Lemma 3 (Leray-Schauder alternative) Let $\mathcal{J}: \mathcal{U} \rightarrow \mathcal{U}$ be a completely continuous operator (i.e., a map that restricted to any bounded set in $\mathcal{U}$ is compact). Let

$$
\mathcal{G}(\mathcal{J})=\{x \in \mathcal{U}: x=\lambda \mathcal{J}(x) \text { for some } 0<\lambda<1\}
$$

Then either the set $\mathcal{G}(\mathcal{J})$ is unbounded, or $\mathcal{J}$ has at lest one fixed point.

Theorem 2 Let $f_{1}, f_{2}, g_{i}, h_{j}:[0,1] \times \mathbb{R} \times \mathbb{R} \rightarrow \mathbb{R}(i=1, \ldots, k)(j=1, \ldots, l)$ be continuous functions such that the following condition holds:

$\left(H_{3}\right)$ There exist real constants $\kappa_{\epsilon}, \hat{\kappa}_{\epsilon}, \rho_{i, \epsilon}, \hat{\rho}_{j, \epsilon} \geq 0(\epsilon=1,2)$ and $\kappa_{0}, \hat{\kappa}_{0}, \rho_{i, 0}, \hat{\rho}_{j, 0}>0$ such that, for $x, y \in \mathbb{R}$,

$$
\begin{array}{ll}
\left|f_{1}(t, x, y)\right| \leq \kappa_{0}+\kappa_{1}|x|+\kappa_{2}|y|, & \left|f_{2}(t, x, y)\right| \leq \hat{\kappa}_{0}+\hat{\kappa}_{1}|x|+\hat{\kappa}_{2}|y|, \\
\left|g_{i}(t, x, y)\right| \leq \rho_{i, 0}+\rho_{i, 1}|x|+\rho_{i, 2}|y|, & \left|h_{j}(t, x, y)\right| \leq \hat{\rho}_{j, 0}+\hat{\rho}_{j, 1}|x|+\hat{\rho}_{j, 2}|y| .
\end{array}
$$

Then, system (1)-(2) has at least one solution on $[0,1]$ provided that

$$
\begin{aligned}
& \left(\varphi_{1}+\vartheta_{1}\right) \kappa_{1}+\left(\varphi_{2}+\vartheta_{2}\right) \hat{\kappa}_{1}+\left(\Omega_{i}+\Theta_{i}\right) \rho_{i, 1}+\left(\widehat{\Omega}_{j}+\widehat{\Theta}_{j}\right) \hat{\rho}_{j, 1}<1, \\
& \left(\varphi_{1}+\vartheta_{1}\right) \kappa_{2}+\left(\varphi_{2}+\vartheta_{2}\right) \hat{\kappa}_{2}+\left(\Omega_{i}+\Theta_{i}\right) \rho_{i, 2}+\left(\widehat{\Omega}_{j}+\widehat{\Theta}_{j}\right) \hat{\rho}_{j, 2}<1,
\end{aligned}
$$

where $\Omega_{i}, \widehat{\Omega}_{j}$ and $\varphi_{\epsilon}, \epsilon=1,2$, are given by (14), and $\Theta_{i}, \widehat{\Theta}_{j}$ and $\vartheta_{\epsilon}, \epsilon=1,2$, are defined by (15).

Proof In the first step, we show that the operator $\mathcal{Q}: \mathcal{S} \times \mathcal{S} \rightarrow \mathcal{S} \times \mathcal{S}$ is completely continuous. Notice that the operator $\mathcal{Q}$ is continuous in view of the continuity of the functions $f_{1}, f_{2}, g_{i}$, and $h_{j}$.

Let $\mathcal{K} \subset \mathcal{S} \times \mathcal{S}$ be bounded. Then, for all $(x, y) \in \mathcal{K}$, there exist constants $\tau_{1}, \tau_{2}, \varpi_{i}$, and $\widehat{\varpi}_{j}$ such that $\left|f_{1}(t, x(t), y(t))\right| \leq \tau_{1},\left|f_{2}(t, x(t), y(t))\right| \leq \tau_{2},\left|g_{i}(t, x(t), y(t))\right| \leq \varpi_{i},\left|h_{j}(t, x(t), y(t))\right| \leq$ 
$\widehat{\varpi}_{j}(i=1, \ldots, k)(j=1, \ldots, l)$. Then, for any $(x, y) \in \mathcal{K}$, we have

$$
\begin{aligned}
& \left\|\mathcal{Q}_{1}(x, y)\right\| \\
& \leq \sup _{t \in[0,1]}\left\{\int_{0}^{t} \frac{(t-s)^{q-1}}{\Gamma(q)}\left|f_{1}(s, x(s), y(s))\right| d s+\sum_{i=1}^{k} \int_{0}^{t} \frac{(t-s)^{q+p_{i}-1}}{\Gamma\left(q+p_{i}\right)}\left|g_{i}(s, x(s), y(s))\right| d s\right. \\
& +\left|a_{1}\right|+\frac{t}{\left|\Lambda_{1}\right|}\left[| \sigma _ { 1 } | \int _ { 0 } ^ { 1 } \left(\frac{(1-s)^{q-1}}{\Gamma(q)}\left|f_{1}(s, x(s), y(s))\right|\right.\right. \\
& \left.+\sum_{i=1}^{k} \frac{(1-s)^{q+p_{i}-1}}{\Gamma\left(q+p_{i}\right)}\left|g_{i}(s, x(s), y(s))\right|\right) d s \\
& +\left|\sigma_{2}\right| \int_{0}^{1}\left(\frac{(1-s)^{q-2}}{\Gamma(q-1)}\left|f_{1}(s, x(s), y(s))\right|+\sum_{i=1}^{k} \frac{(1-s)^{q+p_{i}-2}}{\Gamma\left(q+p_{i}-1\right)}\left|g_{i}(s, x(s), y(s))\right|\right) d s \\
& +\left|\sigma_{3}\right|\left(\int_{0}^{\zeta} \int_{0}^{s} \frac{(s-u)^{\delta-1}}{\Gamma(\delta)}\left|f_{2}(u, x(u), y(u))\right| d u d s\right. \\
& \left.+\sum_{j=1}^{l} \int_{0}^{\zeta} \int_{0}^{s} \frac{(s-u)^{\delta-1}}{\Gamma(\delta)} \int_{0}^{u} \frac{(u-w)^{v_{j}-1}}{\Gamma\left(v_{j}\right)}\left|h_{j}(w, x(w), y(w))\right| d w d u d s\right) \\
& +\left|\sigma_{4}\right| \int_{0}^{\eta_{m}}\left(\frac{\left(\eta_{m}-s\right)^{\delta-1}}{\Gamma(\delta)}\left|f_{2}(s, x(s), y(s))\right|+\sum_{j=1}^{l} \frac{\left(\eta_{m}-s\right)^{\delta+v_{j}-1}}{\Gamma\left(\delta+v_{j}\right)}\left|h_{j}(s, x(s), y(s))\right|\right) d s \\
& +\left|\sigma_{5}\right| \int_{0}^{1}\left(\frac{(1-s)^{\delta-1}}{\Gamma(\delta)}\left|f_{2}(s, x(s), y(s))\right|+\sum_{j=1}^{l} \frac{(1-s)^{\delta+v_{j}-1}}{\Gamma\left(\delta+v_{j}\right)}\left|h_{j}(s, x(s), y(s))\right|\right) d s \\
& +\left|\sigma_{6}\right| \int_{0}^{1}\left(\frac{(1-s)^{\delta-2}}{\Gamma(\delta-1)}\left|f_{2}(s, x(s), y(s))\right|+\sum_{j=1}^{l} \frac{(1-s)^{\delta+v_{j}-2}}{\Gamma\left(\delta+v_{j}-1\right)}\left|h_{j}(s, x(s), y(s))\right|\right) d s \\
& +\left|\sigma_{7}\right|\left(\int_{0}^{\zeta} \int_{0}^{s} \frac{(s-u)^{q-1}}{\Gamma(q)}\left|f_{1}(u, x(u), y(u))\right| d u d s\right. \\
& \left.+\sum_{i=1}^{k} \int_{0}^{\zeta} \int_{0}^{s} \frac{(s-u)^{q-1}}{\Gamma(q)} \int_{0}^{u} \frac{(u-w)^{p_{i}-1}}{\Gamma\left(p_{i}\right)}\left|g_{i}(w, x(w), y(w))\right| d w d u d s\right) \\
& +\left|\sigma_{8}\right| \int_{0}^{\eta_{m}}\left(\frac{\left(\eta_{m}-s\right)^{q-1}}{\Gamma(q)}\left|f_{1}(s, x(s), y(s))\right|\right. \\
& \left.\left.\left.+\sum_{i=1}^{k} \frac{\left(\eta_{m}-s\right)^{q+p_{i}-1}}{\Gamma\left(q+p_{i}\right)}\left|g_{i}(s, x(s), y(s))\right|\right) d s+\left|\Lambda_{2}\right|\right]\right\} \\
& \leq \tau_{1} \sup _{t \in[0,1]}\left\{\left[\frac{t^{q}}{\Gamma(q+1)}+\frac{t}{\left|\Lambda_{1}\right|}\left(\frac{\left|\sigma_{1}\right|}{\Gamma(q+1)}+\frac{\left|\sigma_{2}\right|}{\Gamma(q)}+\frac{\left|\sigma_{7}\right| \zeta^{q+1}}{\Gamma(q+2)}+\frac{\left|\sigma_{8}\right| \eta_{m}^{q}}{\Gamma(q+1)}\right)\right]\right. \\
& +\tau_{2}\left[\frac{t}{\left|\Lambda_{1}\right|}\left(\frac{\left|\sigma_{3}\right| \zeta^{\delta+1}}{\Gamma(\delta+2)}+\frac{\left|\sigma_{4}\right| \eta_{m}^{\delta}}{\Gamma(\delta+1)}+\frac{\left|\sigma_{5}\right|}{\Gamma(\delta+1)}+\frac{\left|\sigma_{6}\right|}{\Gamma(\delta)}\right)\right] \\
& +\sum_{i=1}^{k} \varpi_{i}\left[\frac{t^{q+p_{i}}}{\Gamma\left(q+p_{i}+1\right)}+\frac{t}{\left|\Lambda_{1}\right|}\left(\frac{\left|\sigma_{1}\right|}{\Gamma\left(q+p_{i}+1\right)}+\frac{\left|\sigma_{2}\right|}{\Gamma\left(q+p_{i}\right)}+\frac{\left|\sigma_{7}\right| \zeta^{q+p_{i}+1}}{\Gamma\left(q+p_{i}+2\right)}\right.\right.
\end{aligned}
$$




$$
\begin{gathered}
\left.\left.+\frac{\left|\sigma_{8}\right| \eta_{m}^{q+p_{i}}}{\Gamma\left(q+p_{i}+1\right)}\right)\right]+\sum_{j=1}^{l} \widehat{\varpi}_{j}\left[\frac { t } { | \Lambda _ { 1 } | } \left(\frac{\left|\sigma_{3}\right| \zeta^{\delta+v_{j}+1}}{\Gamma\left(\delta+v_{j}+2\right)}+\frac{\left|\sigma_{4}\right| \eta_{m}^{\delta+v_{j}}}{\Gamma\left(\delta+v_{j}+1\right)}\right.\right. \\
\left.\left.\left.+\frac{\left|\sigma_{5}\right|}{\Gamma\left(\delta+v_{j}+1\right)}+\frac{\left|\sigma_{6}\right|}{\Gamma\left(\delta+v_{j}\right)}\right)\right]+\left(\left|a_{1}\right|+\left|\frac{\Lambda_{2}}{\Lambda_{1}}\right|\right)\right\} \\
=\tau_{1} \varphi_{1}+\tau_{2} \varphi_{2}+\sum_{i=1}^{k} \varpi_{i} \Omega_{i}+\sum_{j=1}^{l} \widehat{\varpi}_{j} \widehat{\Omega}_{j}+\left|a_{1}\right|+\left|\frac{\Lambda_{2}}{\Lambda_{1}}\right|
\end{gathered}
$$

which implies that

$$
\left\|\mathcal{Q}_{1}(x, y)\right\| \leq \tau_{1} \varphi_{1}+\tau_{2} \varphi_{2}+\sum_{i=1}^{k} \varpi_{i} \Omega_{i}+\sum_{j=1}^{l} \widehat{\varpi}_{j} \widehat{\Omega}_{j}+\left|a_{1}\right|+\left|\frac{\Lambda_{2}}{\Lambda_{1}}\right| .
$$

Similarly, we can find that

$$
\left\|\mathcal{Q}_{2}(x, y)\right\| \leq \tau_{1} \vartheta_{1}+\tau_{2} \vartheta_{2}+\sum_{i=1}^{k} \varpi_{i} \Theta_{i}+\sum_{j=1}^{l} \widehat{\varpi}_{j} \widehat{\Theta}_{j}+\left|b_{1}\right|+\left|\frac{\Lambda_{3}}{\Lambda_{1}}\right| .
$$

Consequently, we get

$$
\begin{aligned}
\|\mathcal{Q}(x, y)\| \leq & \left(\varphi_{1}+\vartheta_{1}\right) \tau_{1}+\left(\varphi_{2}+\vartheta_{2}\right) \tau_{2}+\sum_{i=1}^{k}\left(\Omega_{i}+\Theta_{i}\right) \varpi_{i} \\
& +\sum_{j=1}^{l}\left(\widehat{\Omega}_{j}+\widehat{\Theta}_{j}\right) \widehat{\varpi}_{j}+\left|a_{1}\right|+\left|b_{1}\right|+\left|\frac{\Lambda_{2}+\Lambda_{3}}{\Lambda_{1}}\right| .
\end{aligned}
$$

Therefore, the operator $\mathcal{Q}$ is uniformly bounded. Next, we show that $\mathcal{Q}$ is equicontinuous.

Let $t \in[0,1]$ with $t_{2}<t_{1}$. Then we have

$$
\begin{aligned}
\left|\mathcal{Q}_{1}(x, y)\left(t_{1}\right)-\mathcal{Q}_{1}(x, y)\left(t_{2}\right)\right| & \\
\leq & \left|\int_{0}^{t_{2}} \frac{\left(t_{1}-s\right)^{q-1}-\left(t_{2}-s\right)^{q-1}}{\Gamma(q)} f_{1}(s, x(s), y(s)) d s\right|+\left|\int_{t_{2}}^{t_{1}} \frac{\left(t_{1}-s\right)^{q-1}}{\Gamma(q)} f_{1}(s, x(s), y(s)) d s\right| \\
& +\left|\sum_{i=1}^{k} \int_{0}^{t_{2}} \frac{\left(t_{1}-s\right)^{q+p_{i}-1}-\left(t_{2}-s\right)^{q+p_{i}-1}}{\Gamma\left(q+p_{i}\right)} g_{i}(s, x(s), y(s)) d s\right| \\
& +\left|\int_{t_{2}}^{t_{1}} \frac{\left(t_{1}-s\right)^{q+p_{i}-1}}{\Gamma\left(q+p_{i}\right)} g_{i}(s, x(s)) d s\right| \\
& +\frac{\left|t_{1}-t_{2}\right|}{\left|\Lambda_{1}\right|}\left[| \sigma _ { 1 } | \int _ { 0 } ^ { 1 } \left(\frac{(1-s)^{q-1}}{\Gamma(q)}\left|f_{1}(s, x(s), y(s))\right|\right.\right. \\
& \left.+\sum_{i=1}^{k} \frac{(1-s)^{q+p_{i}-1}}{\Gamma\left(q+p_{i}\right)}\left|g_{i}(s, x(s), y(s))\right|\right) d s \\
& +\left|\sigma_{2}\right| \int_{0}^{1}\left(\frac{(1-s)^{q-2}}{\Gamma(q-1)}\left|f_{1}(s, x(s), y(s))\right|+\sum_{i=1}^{k} \frac{(1-s)^{q+p_{i}-2}}{\Gamma\left(q+p_{i}-1\right)}\left|g_{i}(s, x(s), y(s))\right|\right) d s
\end{aligned}
$$




$$
\begin{aligned}
& +\left|\sigma_{3}\right|\left(\int_{0}^{\zeta} \int_{0}^{s} \frac{(s-u)^{\delta-1}}{\Gamma(\delta)}\left|f_{2}(u, x(u), y(u))\right| d u d s+\sum_{j=1}^{l} \int_{0}^{\zeta} \int_{0}^{s} \frac{(s-u)^{\delta-1}}{\Gamma(\delta)}\right. \\
& \left.\times \int_{0}^{u} \frac{(u-w)^{v_{j}-1}}{\Gamma\left(v_{j}\right)}\left|h_{j}(w, x(w), y(w))\right| d w d u d s\right) \\
& +\left|\sigma_{4}\right| \int_{0}^{\eta_{m}}\left(\frac{\left(\eta_{m}-s\right)^{\delta-1}}{\Gamma(\delta)}\left|f_{2}(s, x(s), y(s))\right|\right. \\
& \left.+\sum_{j=1}^{l} \frac{\left(\eta_{m}-s\right)^{\delta+v_{j}-1}}{\Gamma\left(\delta+v_{j}\right)}\left|h_{j}(s, x(s), y(s))\right|\right) d s+\left|\sigma_{5}\right| \int_{0}^{1}\left(\frac{(1-s)^{\delta-1}}{\Gamma(\delta)}\left|f_{2}(s, x(s), y(s))\right|\right. \\
& \left.+\sum_{j=1}^{l} \frac{(1-s)^{\delta+v_{j}-1}}{\Gamma\left(\delta+v_{j}\right)}\left|h_{j}(s, x(s), y(s))\right|\right) d s+\left|\sigma_{6}\right| \int_{0}^{1}\left(\frac{(1-s)^{\delta-2}}{\Gamma(\delta-1)}\left|f_{2}(s, x(s), y(s))\right|\right. \\
& \left.+\sum_{j=1}^{l} \frac{(1-s)^{\delta+v_{j}-2}}{\Gamma\left(\delta+v_{j}-1\right)}\left|h_{j}(s, x(s), y(s))\right|\right) d s+\left|\sigma_{7}\right|\left(\int_{0}^{\zeta} \int_{0}^{s} \frac{(s-u)^{q-1}}{\Gamma(q)}\right. \\
& \times\left|f_{1}(u, x(u), y(u))\right| d u d s+\sum_{i=1}^{k} \int_{0}^{\zeta} \int_{0}^{s} \frac{(s-u)^{q-1}}{\Gamma(q)} \int_{0}^{u} \frac{(u-w)^{p_{i}-1}}{\Gamma\left(p_{i}\right)} \\
& \left.\times\left|g_{i}(w, x(w), y(w))\right| d w d u d s\right)+\left|\sigma_{8}\right| \int_{0}^{\eta_{m}}\left(\frac{\left(\eta_{m}-s\right)^{q-1}}{\Gamma(q)}\left|f_{1}(s, x(s), y(s))\right|\right. \\
& \left.\left.+\sum_{i=1}^{k} \frac{\left(\eta_{m}-s\right)^{q+p_{i}-1}}{\Gamma\left(q+p_{i}\right)}\left|g_{i}(s, x(s), y(s))\right|\right) d s\right] \\
& \leq \tau_{1}\left\{\left[\frac{\left|2\left(t_{1}-t_{2}\right)^{q}\right|+\left|t_{1}^{q}-t_{2}^{q}\right|}{\Gamma(q+1)}+\frac{\left|t_{1}-t_{2}\right|}{\left|\Lambda_{1}\right|}\left(\frac{\left|\sigma_{1}\right|}{\Gamma(q+1)}+\frac{\left|\sigma_{2}\right|}{\Gamma(q)}+\frac{\left|\sigma_{7}\right| \zeta^{q+1}}{\Gamma(q+2)}\right.\right.\right. \\
& \left.\left.+\frac{\left|\sigma_{8}\right| \eta_{m}^{q}}{\Gamma(q+1)}\right)\right]+\tau_{2}\left[\frac{\left|t_{1}-t_{2}\right|}{\left|\Lambda_{1}\right|}\left(\frac{\left|\sigma_{3}\right| \zeta^{\delta+1}}{\Gamma(\delta+2)}+\frac{\left|\sigma_{4}\right| \eta_{m}^{\delta}}{\Gamma(\delta+1)}+\frac{\left|\sigma_{5}\right|}{\Gamma(\delta+1)}+\frac{\left|\sigma_{6}\right|}{\Gamma(\delta)}\right)\right] \\
& +\sum_{i=1}^{k} \varpi_{i}\left[\frac{\left|2\left(t_{1}-t_{2}\right)^{q+p_{i}}\right|+\left|-t_{1}^{q+p_{i}}+t_{2}^{q+p_{i}}\right|}{\Gamma\left(q+p_{i}+1\right)}+\frac{\left|t_{1}-t_{2}\right|}{\left|\Lambda_{1}\right|}\left(\frac{\left|\sigma_{1}\right|}{\Gamma\left(q+p_{i}+1\right)}+\frac{\left|\sigma_{2}\right|}{\Gamma\left(q+p_{i}\right)}\right.\right. \\
& \left.\left.+\frac{\left|\sigma_{7}\right| \zeta^{q+p_{i}+1}}{\Gamma\left(q+p_{i}+2\right)}+\frac{\left|\sigma_{8}\right| \eta_{m}^{q+p_{i}}}{\Gamma\left(q+p_{i}+1\right)}\right)\right] \\
& +\sum_{j=1}^{l} \widehat{\varpi}_{j}\left[\frac { | t _ { 1 } - t _ { 2 } | } { | \Lambda _ { 1 } | } \left(\frac{\left|\sigma_{3}\right| \zeta^{\delta+v_{j}+1}}{\Gamma\left(\delta+v_{j}+2\right)}+\frac{\left|\sigma_{4}\right| \eta_{m}^{\delta+v_{j}}}{\Gamma\left(\delta+v_{j}+1\right)}\right.\right. \\
& \left.\left.\left.+\frac{\left|\sigma_{5}\right|}{\Gamma\left(\delta+v_{j}+1\right)}+\frac{\left|\sigma_{6}\right|}{\Gamma\left(\delta+v_{j}\right)}\right)\right]\right\} \text {. }
\end{aligned}
$$

Clearly, the operator $\mathcal{Q}$ is equicontinuous. Therefore, it follows that the operator $\mathcal{Q}(x, y)$ is completely continuous.

Finally, we show that the set $\mathcal{P}=\{(x, y) \in \mathcal{S} \times \mathcal{S} \mid(x, y)=\lambda \mathcal{Q}(x, y), 0 \leq \lambda \leq 1\}$ is bounded. Let $(x, y) \in \mathcal{P}$, with $(x, y)=\lambda \mathcal{Q}(x, y)$ and for any $t \in[0,1]$, we have

$$
x(t)=\lambda \mathcal{Q}_{1}(x, y)(t), \quad y(t)=\lambda \mathcal{Q}_{2}(x, y)(t) .
$$


In view of condition $\left(H_{3}\right)$, we can find that

$$
\begin{aligned}
|x(t)| \leq & \varphi_{1}\left(\kappa_{0}+\kappa_{1}|x|+\kappa_{2}|y|\right)+\varphi_{2}\left(\hat{\kappa}_{0}+\hat{\kappa}_{1}|x|+\hat{\kappa}_{2}|y|\right) \\
& +\Omega_{i}\left(\rho_{i, 0}+\rho_{i, 1}|x|+\rho_{i, 2}|y|\right)+\widehat{\Omega}_{j}\left(\hat{\rho}_{j, 0}+\hat{\rho}_{j, 1}|x|+\hat{\rho}_{j, 2}|y|\right)
\end{aligned}
$$

and

$$
\begin{aligned}
|y(t)| \leq & \vartheta_{1}\left(\kappa_{0}+\kappa_{1}|x|+\kappa_{2}|y|\right)+\vartheta_{2}\left(\hat{\kappa}_{0}+\hat{\kappa}_{1}|x|+\hat{\kappa}_{2}|y|\right) \\
& +\Theta_{i}\left(\rho_{i, 0}+\rho_{i, 1}|x|+\rho_{i, 2}|y|\right)+\widehat{\Theta}_{j}\left(\hat{\rho}_{j, 0}+\hat{\rho}_{j, 1}|x|+\hat{\rho}_{j, 2}|y|\right) .
\end{aligned}
$$

Hence

$$
\begin{aligned}
\|x\| \leq & \varphi_{1} \kappa_{0}+\varphi_{2} \hat{\kappa}_{0}+\Omega_{i} \rho_{i, 0}+\widehat{\Omega}_{j} \hat{\rho}_{j, 0}+\left(\varphi_{1} \kappa_{1}+\varphi_{2} \hat{\kappa}_{1}+\Omega_{i} \rho_{i, 1}+\widehat{\Omega}_{j} \hat{\rho}_{j, 1}\right)\|x\| \\
& +\left(\varphi_{1} \kappa_{2}+\varphi_{2} \hat{\kappa}_{2}+\Omega_{i} \rho_{i, 2}+\widehat{\Omega}_{j} \hat{\rho}_{j, 2}\right)\|y\|
\end{aligned}
$$

and

$$
\begin{aligned}
\|y\| \leq & \vartheta_{1} \kappa_{0}+\vartheta_{2} \hat{\kappa}_{0}+\Theta_{i} \rho_{i, 0}+\widehat{\Theta}_{j} \hat{\rho}_{j, 0}+\left(\vartheta_{1} \kappa_{1}+\vartheta_{2} \hat{\kappa}_{1}+\Theta_{i} \rho_{i, 1}+\widehat{\Theta}_{j} \hat{\rho}_{j, 1}\right)\|x\| \\
& +\left(\vartheta_{1} \kappa_{2}+\vartheta_{2} \hat{\kappa}_{2}+\Theta_{i} \rho_{i, 2}+\widehat{\Theta}_{j} \hat{\rho}_{j, 2}\right)\|y\| .
\end{aligned}
$$

In consequence, we get

$$
\begin{aligned}
\|x\|+\|y\| \leq & \left(\varphi_{1}+\vartheta_{1}\right) \kappa_{0}+\left(\varphi_{2}+\vartheta_{2}\right) \hat{\kappa}_{0}+\left(\Omega_{i}+\Theta_{i}\right) \rho_{i, 0}+\left(\widehat{\Omega}_{j}+\widehat{\Theta}_{j}\right) \hat{\rho}_{j, 0} \\
& +\left(\left(\varphi_{1}+\vartheta_{1}\right) \kappa_{1}+\left(\varphi_{2}+\vartheta_{2}\right) \hat{\kappa}_{1}+\left(\Omega_{i}+\Theta_{i}\right) \rho_{i, 1}+\left(\widehat{\Omega}_{j}+\widehat{\Theta}_{j}\right) \hat{\rho}_{j, 1}\right)\|x\| \\
& +\left(\left(\varphi_{1}+\vartheta_{1}\right) \kappa_{2}+\left(\varphi_{2}+\vartheta_{2}\right) \hat{\kappa}_{2}+\left(\Omega_{i}+\Theta_{i}\right) \rho_{i, 2}+\left(\widehat{\Omega}_{j}+\widehat{\Theta}_{j}\right) \hat{\rho}_{j, 2}\right)\|y\|,
\end{aligned}
$$

which implies that

$$
\|(x, y)\| \leq \frac{\left(\varphi_{1}+\vartheta_{1}\right) \kappa_{0}+\left(\varphi_{2}+\vartheta_{2}\right) \hat{\kappa}_{0}+\left(\Omega_{i}+\Theta_{i}\right) \rho_{i, 0}+\left(\widehat{\Omega}_{j}+\widehat{\Theta}_{j}\right) \hat{\rho}_{j, 0}}{E_{0}},
$$

where

$$
\begin{aligned}
E_{0}= & \min \left\{1-\left(\left(\varphi_{1}+\vartheta_{1}\right) \kappa_{1}+\left(\varphi_{2}+\vartheta_{2}\right) \hat{\kappa}_{1}+\left(\Omega_{i}+\Theta_{i}\right) \rho_{i, 1}+\left(\widehat{\Omega}_{j}+\widehat{\Theta}_{j}\right) \hat{\rho}_{j, 1}\right),\right. \\
& \left.1-\left(\left(\varphi_{1}+\vartheta_{1}\right) \kappa_{2}+\left(\varphi_{2}+\vartheta_{2}\right) \hat{\kappa}_{2}+\left(\Omega_{i}+\Theta_{i}\right) \rho_{i, 2}+\left(\widehat{\Omega}_{j}+\widehat{\Theta}_{j}\right) \hat{\rho}_{j, 2}\right)\right\} .
\end{aligned}
$$

Hence the set $\mathcal{P}$ is bounded. Thus, by Lemma 3, the operator $\mathcal{Q}$ has at least one fixed point, which implies that system (1)-(2) has at least one solution on $[0,1]$.

\section{Examples}

Here, we present illustrative examples for the results proved in the last section. 
Example 1 Consider the following system:

$$
\begin{cases}{ }^{c} D^{3 / 2} x(t)+\sum_{i=1}^{2} I^{p_{i}} g_{i}(t, x(t), y(t))=f_{1}(t, x(t), y(t)), & 1<q \leq 2, t \in[0,1], \\ { }^{c} D^{5 / 4} y(t)+\sum_{j=1}^{2} I^{v_{j}} h_{j}(t, x(t), y(t))=f_{2}(t, x(t), y(t)), & 1<\delta \leq 2, t \in[0,1]\end{cases}
$$

complemented with the boundary conditions

$$
\left\{\begin{array}{l}
x(0)=1, \quad y(0)=3, \\
\alpha_{1} x(1)+\beta_{1} x^{\prime}(1)=\gamma_{1} \int_{0}^{\zeta} y(s) d s+\sum_{m=1}^{3} \mu_{m} y\left(\eta_{m}\right), \\
\alpha_{2} y(1)+\beta_{2} y^{\prime}(1)=\gamma_{2} \int_{0}^{\zeta} x(s) d s+\sum_{m=1}^{3} \xi_{m} x\left(\eta_{m}\right) .
\end{array}\right.
$$

Here, $\alpha_{1}=1 / 4, \alpha_{2}=1 / 2, \beta_{1}=3 / 5, \beta_{2}=4 / 5, \gamma_{1}=\gamma_{2}=1, \zeta=1 / 7 \eta_{1}=1 / 5, \eta_{2}=2 / 5, \eta_{3}=3 / 5$, $\mu_{1}=1 / 2, \mu_{2}=3 / 4, \mu_{3}=1, \xi_{1}=1 / 3, \xi_{2}=2 / 3, \xi_{3}=1$, and

$$
\begin{aligned}
& f_{1}(t, x(t), y(t))=\frac{1}{18 \sqrt{169+t^{4}}}\left(\frac{|x(t)|}{1+|x(t)|}+\tan ^{-1} y(t)\right)+\cos 2 t, \\
& f_{2}(t, x(t), y(t))=\frac{1}{8\left(t^{2}+30\right)} \sin x(t)+\frac{\tan ^{-1} y(t)}{12 \sqrt{t^{2}+400}}+\frac{1}{24+t^{2}}, \\
& g_{1}(t, x(t), y(t))=\frac{1}{\left(118+t^{2}\right)}(\sin x(t)+|y(t)|)+3 t \\
& g_{2}(t, x(t), y(t))=\frac{1}{9 \sqrt{196+t^{5}}}(x(t)+\cos y(t))+3 e^{-t}, \\
& h_{1}(t, x(t), y(t))=\frac{1}{6 \sqrt{289+t^{2}}}\left(x(t)+\tan ^{-1} x(t)\right)+\frac{1}{\sqrt{4}+100+t^{2}}\left(\frac{|y(t)|}{1+|y(t)|}+\sin 2 t\right), \\
& h_{2}(t, x(t), y(t))=\frac{e^{-t}}{3\left(4+t^{2}\right)}\left(\cos t+\tan ^{-1} x(t)\right)+\frac{e^{-t} \sqrt{9}}{3\left(t^{2}+12\right)}(\sin y(t)+5 t) .
\end{aligned}
$$

Clearly, we have

$$
\begin{aligned}
\left|f_{1}\left(t, x_{1}, y_{1}\right)-f_{1}\left(t, x_{2}, y_{2}\right)\right| & \leq \frac{1}{234}\left(\left|x_{1}-y_{1}\right|+\left|y_{1}-y_{2}\right|\right), \\
\left|f_{2}\left(t, x_{1}, y_{1}\right)-f_{2}\left(t, x_{2}, y_{2}\right)\right| & \leq \frac{1}{240}\left(\left|x_{1}-y_{1}\right|+\left|y_{1}-y_{2}\right|\right), \\
\left|g_{1}\left(t, x_{1}, y_{1}\right)-g_{1}\left(t, x_{2}, y_{2}\right)\right| & \leq \frac{1}{118}\left(\left|x_{1}-y_{1}\right|+\left|y_{1}-y_{2}\right|\right), \\
\left|g_{2}\left(t, x_{1}, y_{1}\right)-g_{2}\left(t, x_{2}, y_{2}\right)\right| & \leq \frac{1}{126}\left(\left|x_{1}-y_{1}\right|+\left|y_{1}-y_{2}\right|\right), \\
\left|h_{1}\left(t, x_{1}, y_{1}\right)-h_{1}\left(t, x_{2}, y_{2}\right)\right| & \leq \frac{1}{102}\left(\left|x_{1}-y_{1}\right|+\left|y_{1}-y_{2}\right|\right), \\
\left|h_{2}\left(t, x_{1}, y_{1}\right)-h_{2}\left(t, x_{2}, y_{2}\right)\right| & \leq \frac{1}{12 e}\left(\left|x_{1}-y_{1}\right|+\left|y_{1}-y_{2}\right|\right) .
\end{aligned}
$$

Using the given data, we have $\Lambda_{1}=0.151835$ and $\Psi=0.788452<1$. Obviously the hypotheses of Theorem 1 are satisfied. Hence, by the conclusion of Theorem 1 , there is a unique solution for problem (18)-(19) on $[0,1]$. 
Example 2 Consider problem (18)-(19) with the following data:

$$
\begin{aligned}
& f_{1}(t, x(t), y(t))=\frac{3 \sin t}{\left(145+t^{5}\right)}+\frac{1}{158} x(t) \cos y(t)+\frac{e^{-t}}{3 \sqrt{169}} \tan ^{-1} y(t) \\
& f_{2}(t, x(t), y(t))=\frac{2}{13 \sqrt{49+t}}+\frac{\sin x(t)}{11 \sqrt{144+t^{2}}}+\frac{y(t)}{165 t^{3}} \\
& g_{1}(t, x(t), y(t))=e^{-15 t}+\frac{1}{124} x(t) \tan ^{-1} y(t)+\frac{y(t)}{14 \sqrt{81+t^{3}}} \\
& g_{2}(t, x(t), y(t))=\frac{2}{17 \sqrt{81+t^{2}}}+\frac{1}{\left(113+t^{2}\right)} x(t)+\frac{e^{-t}}{280} \sin y(t), \\
& h_{1}(t, x(t), y(t))=\frac{3}{82 \sqrt{t}}+\frac{e^{-t}}{38 t^{2}} \cos x(t)+\frac{1}{8 \sqrt{144+t^{5}} y(t),} \\
& h_{2}(t, x(t), y(t))=e^{-13 t}+\frac{3}{148} x(t) \tan ^{-1} y(t)+\frac{y(t)}{11 \sqrt{t^{2}+81}} .
\end{aligned}
$$

It is easy to check that condition $\left(H_{3}\right)$ is satisfied with $\kappa_{0}=3 / 146, \kappa_{1}=1 / 158, \kappa_{2}=1 / 39 e$, $\hat{\kappa}_{0}=2 / 91, \hat{\kappa}_{1}=1 / 132, \hat{\kappa}_{2}=1 / 165, \rho_{1,0}=1 / 15 e, \rho_{1,1}=1 / 124, \rho_{1,2}=1 / 126, \rho_{2,0}=2 / 153, \rho_{2,1}=$ $1 / 113, \rho_{2,2}=1 / 280, \hat{\rho}_{1,0}=3 / 82, \hat{\rho}_{1,1}=1 / 38 e, \hat{\rho}_{1,2}=1 / 96, \hat{\rho}_{1,0}=1 / 13 e, \hat{\rho}_{2,1}=3 / 148, \hat{\rho}_{2,2}=$ 1/99. Furthermore, $\left(\varphi_{1}+\vartheta_{1}\right) \kappa_{1}+\left(\varphi_{2}+\vartheta_{2}\right) \hat{\kappa}_{1}+\left(\Omega_{i}+\Theta_{i}\right) \rho_{i, 1}+\left(\widehat{\Omega}_{j}+\widehat{\Theta}_{j}\right) \hat{\rho}_{j, 1} \approx 0.948955<1$, and $\left(\varphi_{1}+\vartheta_{1}\right) \kappa_{2}+\left(\varphi_{2}+\vartheta_{2}\right) \hat{\kappa}_{2}+\left(\Omega_{i}+\Theta_{i}\right) \rho_{i, 2}+\left(\widehat{\Omega}_{j}+\widehat{\Theta}_{j}\right) \hat{\rho}_{j, 2} \approx 0.846541<1$. Therefore the hypotheses of Theorem 2 are satisfied. Hence, by the conclusion of Theorem 2, problem (18)-(19) with data (20) has at least one solution on $[0,1]$.

\section{Conclusions}

Existence and uniqueness results are derived for a system of nonlinear coupled CaputoRiemann-Liouville type fractional integro-differential equations equipped with multipoint sub-strip boundary conditions. Our results are not only new in the given configuration, but also yield some new results associated with particular choices of the parameters involved in the problem at hand. For example, our results correspond to a system of nonlinear coupled Caputo-Riemann-Liouville type fractional integro-differential equations equipped with coupled multi-point boundary conditions if we take $\gamma_{1}=0=\gamma_{2}$ in the results of this paper. In case we take $\mu_{m}=0=\xi_{m}$ for all $m=1,2, \ldots, \omega$, we get the results for a coupled system of nonlinear Caputo-Riemann-Liouville type fractional integrodifferential equations supplemented with coupled sub-strip boundary conditions.

Acknowledgements

The Deanship of Scientific Research (DSR) at King Abdulaziz University, Jeddah, Saudi Arabia funded this project, under grant no. FP-19-42.

Funding

The Deanship of Scientific Research (DSR) at King Abdulaziz University, Jeddah, Saudi Arabia funded this project, under grant no. FP-19-42.

\section{Abbreviations}

Not applicable.

Availability of data and materials

Not applicable. 
Authors' contributions

Each of the authors, AA, AFA, SKN, and BA contributed equally to each part of this work. All authors read and approved the final manuscript.

\section{Author details}

${ }^{1}$ Nonlinear Analysis and Applied Mathematics (NAAM) - Research Group, Department of Mathematics, Faculty of Science, King Abdulaziz University, P.O. Box 80203, Jeddah 21589, Saudi Arabia. ${ }^{2}$ Department of Mathematics, University of loannina, 45110 loannina, Greece.

\section{Publisher's Note}

Springer Nature remains neutral with regard to jurisdictional claims in published maps and institutional affiliations.

Received: 23 September 2020 Accepted: 10 December 2020 Published online: 07 January 2021

\section{References}

1. Magin, R.L.: Fractional Calculus in Bioengineering. Begell House Publishers, Danbury (2006)

2. Javidi, M., Ahmad, B.: Dynamic analysis of time fractional order phytoplankton-toxic phytoplankton-zooplankton system. Ecol. Model. 318, 8-18 (2015)

3. Fallahgoul, H.A., Focardi, S.M., Fabozzi, F.J.: Fractional Calculus and Fractional Processes with Applications to Financial Economics. Theory and Application. Elsevier/Academic Press, London (2017)

4. Zaslavsky, G.M.: Hamiltonian Chaos and Fractional Dynamics. Oxford University Press, Oxford (2005)

5. Javidi, M., Ahmad, B.: Dynamic analysis of time fractional order phytoplankton-toxic phytoplankton-zooplankton system. Ecol. Model. 318, 8-18 (2015)

6. Carvalho, A., Pinto, C.M.A.: A delay fractional order model for the co-infection of malaria and HIV/AIDS. Int. J. Dyn. Control 5, 168-186 (2017)

7. Yu, N., Zhu, W.: Event-triggered impulsive chaotic synchronization of fractional-order differential systems. Appl. Math. Comput. 388, $125554(2021)$

8. Henderson, J., Luca, R., Tudorache, A.: On a system of fractional differential equations with coupled integral boundary conditions. Fract. Calc. Appl. Anal. 18, 361-386 (2015)

9. Ahmad, B., Luca, R.: Existence of solutions for sequential fractional integro-differential equations and inclusions with nonlocal boundary conditions. Appl. Math. Comput. 339, 516-534 (2018)

10. Ahmad, B., Alsaedi, A., Aljoudi, S., Ntouyas, S.K.: A six-point nonlocal boundary value problem of nonlinear coupled sequential fractional integro-differential equations and coupled integral boundary conditions. J. Appl. Math. Comput. 56, 367-389 (2018)

11. Alsaedi, A., Ahmad, B., Aljoudi, S., Ntouyas, S.K.: A study of a fully coupled two-parameter system of sequential fractional integro-differential equations with nonlocal integro-multipoint boundary conditions. Acta Math. Sci. Ser. B Engl. Ed. 39, 927-944 (2019)

12. Feckan, M., Marynets, K., Wang, J.R.: Periodic boundary value problems for higher-order fractional differential systems. Math. Methods Appl. Sci. 42, 3616-3632 (2019)

13. Guendouz, C., Lazreg, J.E., Nieto, J.J., Ouahab, A.: Existence and compactness results for a system of fractional differential equations. J. Funct. Spaces 2020, Article ID 5735140 (2020)

14. Wongcharoen, A., Ntouyas, S.K., Tariboon, J.: On coupled systems for Hilfer fractional differential equations with nonlocal integral boundary conditions. J. Math. 2020, Article ID 2875152 (2020)

15. Ahmad, B., Alghamdi, N., Alsaedi, A., Ntouyas, S.K.: A system of coupled multi-term fractional differential equations with three-point coupled boundary conditions. Fract. Calc. Appl. Anal. 22,601-618 (2019)

16. Alsaedi, A., Ahmad, B., Aljoudi, S., Ntouyas, S.K.: A study of a fully coupled two-parameter system of sequential fractional integro-differential equations with nonlocal integro-multipoint boundary conditions. Acta Math. Sci. Ser. B Engl. Ed. 39, 927-944 (2019)

17. Zhou, Y., Suganya, S., Arjunan, M.M., Ahmad, B.: Approximate controllability of impulsive fractional integro-differential equation with state-dependent delay in Hilbert spaces. IMA J. Math. Control Inf. 36, 603-622 (2019)

18. Liu, J., Zhao, K.: Existence of mild solution for a class of coupled systems of neutral fractional integro-differential equations with infinite delay in Banach space. Adv. Differ. Equ. 2019, 284 (2019)

19. Ravichandran, C., Valliammal, N., Nieto, J.J.: New results on exact controllability of a class of fractional neutral integro-differential systems with state-dependent delay in Banach spaces. J. Franklin Inst. 356, 1535-1565 (2019)

20. Ahmad, B., Alsaedi, A., Ntouyas, S.K.: Fractional order nonlinear mixed coupled systems with coupled integro-differential boundary conditions. J. Appl. Anal. Comput. 10, 892-903 (2020)

21. Ntouyas, S.K., Al-Sulami, H.H.: A study of coupled systems of mixed order fractional differential equations and inclusions with coupled integral fractional boundary conditions. Adv. Differ. Equ. 2020, 73 (2020)

22. Kilbas, A.A., Srivastava, H.M., Trujillo, J.J.: Theory and Applications of Fractional Differential Equations. North-Holland Mathematics Studies, vol. 204. Elsevier, Amsterdam (2006)

23. Granas, A., Dugundji, J.: Fixed Point Theory. Springer, New York (2005) 